\title{
Magnetocrystalline anisotropy energy of Co and Fe adatoms on the (111) surfaces of Pd and Rh
}

\author{
Piotr Błoński, ${ }^{1}$ Anne Lehnert, ${ }^{2}$ Samuel Dennler, ${ }^{3}$ Stefano Rusponi, ${ }^{2}$ Markus Etzkorn, ${ }^{2}$ Géraud Moulas, ${ }^{2}$ Peter Bencok, ${ }^{4}$ \\ Pietro Gambardella, ${ }^{5,6}$ Harald Brune, ${ }^{2}$ and Jürgen Hafner ${ }^{1}$ \\ ${ }^{1}$ Fakultät für Physik and Center for Computational Materials Science, Universität Wien, Sensengasse 8/12, A-1090 Wien, Austria \\ ${ }^{2}$ Institute of Condensed Matter Physics, Ecole Polytechnique Fédérale de Lausanne (EPFL), CH-1015 Lausanne, Switzerland \\ ${ }^{3}$ Laboratoire des Collö̈des, Verres et Nanomatériaux, Université de Montpellier II, F-34095 Montpellier, France \\ ${ }^{4}$ European Synchrotron Radiation Facility, Boîte Postale 200, F-38043 Grenoble, France \\ 5 Institució Catalana de Recerca i Estudis Avançats (ICREA), E-08100 Barcelona, Spain \\ ${ }^{6}$ Centre d'Investigació en Nanociència i Nanotecnologia (ICN-CSIC), UAB Campus, E-08193 Bellaterra, Spain
}

(Received 24 November 2009; revised manuscript received 8 March 2010; published 30 March 2010)

\begin{abstract}
We performed a combined theoretical and experimental investigation of the orbital magnetism and magnetocrystalline anisotropy of isolated $\mathrm{Co}$ and $\mathrm{Fe}$ adatoms on $\mathrm{Pd}(111)$ and $\mathrm{Rh}(111)$. Theoretical calculations of the spin and orbital moments are based on ab initio spin-polarized density-functional theory (DFT) including a self-consistent treatment of spin-orbit coupling. The calculations use a slab model to represent the adsorbate/ substrate complex and allow for a complete structural relaxation leading to a strong inward displacement of the adatom and modest vertical and lateral relaxations in the substrate atoms. Compared to an idealized geometry where the atoms are kept on bulk lattice positions up to the surface, relaxation leads to a much stronger adatom/ligand hybridization. This is also reflected in the results for orbital moments and magnetocrystalline anisotropy energy (MAE). The enhanced hybridization leads to strong quenching of the adatom orbital moments but also to the formation of large induced spin and orbital moments in the substrate. As a consequence, we find that the substrate contribution to the MAE is much more important than estimated before on the basis of studies using an idealized geometry. We also find the surprising result that the MAE strongly depends on the adsorption site. The magnitude and even the sign of the MAE change for adatoms on face-centered cubic with respect to the ones on hexagonal close-packed hollow sites on the (111) surface. The dependence of the MAE on the combination of adatom and substrate has been analyzed in terms of the electronic structure, leading to a sound physical picture of the origin of the MAE. A fundamental problem, however, is the correct prediction of the size of the orbital moments of the adatoms. We suggest that this problem can be solved only via post-DFT corrections introducing an orbital dependence of the exchange potential. The theoretical results are compared to site-averaged, element-specific x-ray magnetic circular dichroism (XMCD) measurements. Lowtemperature XMCD spectra and magnetization curves reveal weak out-of-plane anisotropy for Fe adatoms on both substrates. Interestingly, Co adatoms on $\mathrm{Rh}(111)$ present in-plane anisotropy with MAE of about $-0.6 \mathrm{meV}$, contrary to the known out-of-plane anisotropy of Co on $\mathrm{Pd}(111)$ and $\mathrm{Pt}(111)$. The orbital to spin magnetic-moment ratio measured by XMCD shows that the Co adatoms present much stronger orbital magnetization components compared to Fe. The connection between orbital moments and MAE is discussed at the theoretical level including the contribution of the induced substrate magnetization.
\end{abstract}

DOI: $10.1103 /$ PhysRevB.81.104426

PACS number(s): 75.30.Gw, 75.75.-c, 71.15.Mb, 78.70.Dm

\section{INTRODUCTION}

The desire to build magnetic or magneto-optical storage devices with maximal storage density ${ }^{1,2}$ has spurred a strong interest in the properties of magnetic nanostructures supported on nonmagnetic substrates. ${ }^{3}$ One bit of information is stored per magnetic domain, and a high magnetic anisotropy is required to inhibit magnetization reversal and hence loss of information. A reduction in the size of the nanostructure carrying one bit of information requires the increase in the magnetic anisotropy energy (MAE) per atom. In addition, the easy axis must be oriented perpendicular to the surface of the substrate to reduce the dipolar magnetic interactions between neighboring magnetic moments. The ultimate size limit of a system showing magnetic anisotropy is an isolated magnetic atom on a nonmagnetic substrate. A high MAE requires large spin and orbital moments and a strong spin-orbit coupling (SOC). Recently, a giant MAE was found by Gambardella et $a l{ }^{4}$ for Co atoms on a $\operatorname{Pt}(111)$ substrate. In this case, the large magnetic moment is provided by the Co atom but for Co, like for all $3 d$ ferromagnets, the spin-orbit coupling is weak. Strong SOC is found in the heavy $5 d$ metals such as $\mathrm{Pt}$ but these metals are nonmagnetic. Hence the giant MAE observed for $\mathrm{Co} / \mathrm{Pt}(111)$ must have its origin in the strong coupling between the magnetic adatom and its ligands. The situation is similar as for ultrathin films of $3 d$ elements adsorbed on heavy-metal substrates or multilayers formed by these systems. ${ }^{5}$

The investigation of the MAE of supported magnetic nanostructures remains a challenge to both theory and experiment. Using X-ray magnetic circular dichroism (XMCD) (Ref. 6), it is now possible to measure the magnetic properties of submonolayers down to coverages as low as 0.002 monolayers (MLs). ${ }^{7}$ At these coverages statistical growth, obtained by deposition at temperatures where monomer diffusion is frozen, leads almost exclusively to single adatoms. ${ }^{8}$ Due to the spatial extent of the x-ray beam of a few hundred micrometer square, XMCD performs ensemble averages. 
However, for ensembles containing only monomers with no mutual magnetic interactions, XMCD enables one to measure the spin and orbital moments as well as the MAE of single atoms. A review of experimental investigations of the magnetic properties of isolated atoms adsorbed on surfaces has recently been presented by Brune and Gambardella. ${ }^{9}$ The results for $\mathrm{Co} / \mathrm{Pt}(111)$ remain characteristic for the magnetism of $3 d$ atoms on $4 d$ or $5 d$ substrates. Both spin and orbital moment of the Co atoms $\left(\mu_{S}=1.8 \pm 0.1 \mu_{B}, \mu_{L}\right.$ $\left.=1.1 \pm 0.1 \mu_{B}\right)$ measured at low temperature and in a magnetic field strong enough to achieve saturation are strongly enhanced compared not only to bulk Co (where $\mu_{S}$ $\left.=1.52 \mu_{B}, \mu_{L}=0.15 \mu_{B}\right)$ but also in comparison to ultrathin films, ${ }^{5,10}$ atomic wires, ${ }^{11,12}$ and supported nanoclusters. ${ }^{10,13}$ From the magnetization curves measured in magnetic fields perpendicular and nearly parallel to the surface, a very large MAE of $9.3 \pm 1.6 \mathrm{meV}$ and a total moment per Co atom (measuring the sum of the spin and orbital moments of the magnetic adatom plus the magnetization induced in the substrate) of $5.0 \pm 0.6 \mu_{B}$ have been estimated. A similar "giant effective moment" of about $10 \mu_{B} /$ Co atom has been reported for very dilute Co-Pd alloys. ${ }^{14}$ Considering the difference in the coordination between an adatom adsorbed on a surface and an atom immersed in the bulk, both estimates are in reasonable agreement.

Additional information on the structural and magnetic properties of $\mathrm{Co}$ atoms adsorbed on $\mathrm{Pt}(111)$ comes from scanning tunneling microscopy (STM) and scanning tunneling spectroscopy (STS). ${ }^{15,16}$ The STM images confirm that the Co atoms occupy both face-centered cubic (fcc) and hexagonal close-packed (hcp) hollows of the Pt surface. The particular advantage of spin-polarized STS measurements is that they allow to probe the magnetic properties of individual adatoms. ${ }^{16}$ Assuming a perpendicular easy axis of magnetization and a MAE of $9.3 \mathrm{meV}$ taken from the XMCD experiments, a fit of the magnetization curves as a function of the applied field yields slightly different total effective moments of $\mu(\mathrm{hcp})=3.9 \pm 0.2 \mu_{B}$ and $\mu(\mathrm{fcc})=3.5 \pm 0.2 \mu_{B}$ for Co atoms in the two different adsorption sites. Inelastic tunneling spectroscopy observes the exchange of spin angular momentum between the tunneling electrons. ${ }^{17,18}$ Very recently, it has been proposed that the MAE may be derived from the spin-flip energy. ${ }^{19-21}$ However, to derive the MAE from the spin-excitation energy, the magnitude of the spin $S$ to be flipped (consisting of the spins of the adsorbed atom plus the induced substrate moments) must be known. If $S$ $>1$, the excitation from the ground state with $S_{z}= \pm S$ to a state with $S_{z}= \pm(S-1)$ does not necessarily correspond to a spin reorientation by $90^{\circ}$. For $\mathrm{Co} / \mathrm{Pt}(111)$, Balashov et al. ${ }^{21}$ assumed a spin of $S=1$ to derive an MAE compatible with the XMCD result. For Fe/Pt(111), a similar large MAE of 6.5 meV was derived from the STS data, assuming a spin of $S$ $=3 / 2$.

The determination of the spin and orbital moments and of the MAE of supported magnetic nanostructures also represents a challenge to current spin-density-functional theories (SDFTs) of magnetism. Magnetic anisotropy is caused by spin-orbit coupling, hence a relativistic calculation is required. The essential problem, however, is twofold: (i) the strong coupling between nanostructure and substrate must be correctly described. This is particularly important for isolated adatoms. The spin degeneracy of the free atom is broken only by the coupling to the ligands. (ii) All current versions of SDFT tend to underestimate the orbital moment. The reason is that the charge and spin densities determining the effective one-electron potential are determined as averages over occupied orbitals. It has been proposed that the evaluation of the orbital moments could be improved by adding semiempirical corrections such as the orbital-polarization term proposed by Brooks et al. ${ }^{22}$ However, calculations based on the full orbital-polarization term produce too large moments. $^{4,23}$

In any calculation of the MAE, a number of technical choices are required: (i) choice of the exchange-correlation functional: local or semilocal (gradient-corrected), eventually adding post SDFT corrections. (ii) Self-consistent bandstructure or Green's-function calculations - this also implies the choice of the form of the one-electron potential (fullpotential or atomic-sphere approximation). (iii) Spin-orbit coupling taken into account by solving the Dirac equation 24,25 or by using the second-order variational approach. $^{26,27}$ (iii) Modeling of the substrate-periodic slabs or semi-infinite (possible only within Green's-function approaches). (iv) Geometry of the adsorbate/substrate complex-all atoms in sites continuing the crystalline lattice of the bulk substrate, relaxation of the adatom only, or full relaxation of adatom plus substrate. (v) MAE determined in terms of independent total-energy calculations for different orientations of the magnetization or using the "magnetic force theorem (FT)" 28,29 as the difference in the band energies at a frozen potential and charge density. Very recently, we have published a study attempting to assess the influence of these different approximations on ab initio calculations of the MAE. ${ }^{30}$

For the archetypal systems $\mathrm{Co} / \mathrm{Pt}(111)$ and $\mathrm{Fe} / \mathrm{Pt}(111)$, a number of SDFT studies of orbital moments and MAE have been published. , $21,30-35$ With the exception of our recent work $^{30}$ and the work by Shick and Lichtenstein ${ }^{35}$ and by Balashov et al., ${ }^{21}$ all these calculations are based on a Green's-function approach within the screened fully relativistic Kohn-Korringa-Rostocker (KKR) method, an idealized geometry propagating the bulk structure of the substrate, the local-density approximation, and the use of the magnetic force theorem. ${ }^{4,21,31-35}$ In our recent assessment of the impact of the different approximations, we have demonstrated that the most important step is the description of the adsorbate/ substrate complex. Calculations based on the assumption of an idealized bulklike geometry essentially attempt to cure a defect of the SDFT (missing orbital dependence of the exchange field) by artificially minimizing adatom/support hybridization. This allows to achieve a larger orbital moment and an MAE in semiquantitative agreement with experiment at the expense of an unrealistic geometry and electronic structure. Calculations based on a relaxed geometry provide a more realistic scenario but tend to underestimate the orbital moments even more strongly. Hence both types of calculations provide rather a lower limit to the MAE: in one case the influence of the strong SOC of the ligands and in the other the orbital moments of the adatom is underestimated.

In the present work, we report on a combined theoretical and experimental investigation of the spin and orbital mo- 
ments as well as the MAE of isolated Co and Fe atoms on $\mathrm{Pd}(111)$ and $\mathrm{Rh}(111)$ substrates. Compared to the wellstudied $\mathrm{Co}(\mathrm{Fe}) / \mathrm{Pt}(111)$ case, these systems based on $4 d$ substrates differ by a reduced strength of the SOC but an increased magnetic polarizability of the substrate metals. Both $\mathrm{Pd}$ and $\mathrm{Rh}$ are known to be nearly magnetic, their density of states at the Fermi level just fails to meet the Stoner limit for ferromagnetic ordering. For both metals, there have been reports that at least their more open (100) surfaces are magnetic. This has led to a lengthy and heated discussion but the question seems now to be settled in favor of the absence of surface magnetism. Nonetheless, due to the high polarizability of the $\mathrm{Rh}$ atom, for $\mathrm{Rh}$ nanostructures (monolayers on $\mathrm{Ag}$ or $\mathrm{Au}$ substrates, ${ }^{36,37} \mathrm{Rh}$ nanowires, ${ }^{38}$ and free $\mathrm{Rh}$ clusters ${ }^{39,40}$ ), the existence of a spontaneous magnetization has been predicted, even though not verified experimentally. ${ }^{41}$ Experimental confirmation for the magnetism of free $\mathrm{Rh}$ clusters is available from Stern-Gerlach experiments. ${ }^{42}$ For Fe/Pd, a "giant" enhancement of the magnetic moment of Fe impurities, ${ }^{43,44}$ of embedded Fe monolayers and artificial layered FePt and FePd compounds with an $\mathrm{L}_{0}$ structure has been reported. ${ }^{45}$ The recent SDFT studies of $\mathrm{Rh}$ surfaces magnetically doped with Co impurities ${ }^{37}$ have demonstrated that the induced spin moments on $\mathrm{Rh}$ atoms may be as large as $0.7 \mu_{B}$ and that the total moment per Co atom may reach $4 \mu_{B}$. However, these studies have also demonstrated that the magnetic polarization induced by a Ruderman-Kittel-Kasuya-Yoshida mechanism is long ranged and that convergence with respect to the model size may be difficult to achieve.

\section{COMPUTATIONAL SETUP}

$A b$ initio density-functional calculations of the spin and orbital magnetic moments and of the magnetic anisotropy energy of $\mathrm{Fe}$ and $\mathrm{Co}$ adatoms on $\mathrm{Rh}$ and $\mathrm{Pd}$ (111) surfaces were performed using the Vienna $a b$ initio simulation package (VASP). ${ }^{46}$ VASP performs an iterative solution of the Kohn-Sham equations within a plane-wave basis. Electronic exchange and correlation are described within a spinpolarized generalized-gradient approximation (GGA) using the functional proposed by Perdew et al. (PW91 - Ref. 47) and the spin interpolation proposed by Vosko et al. ${ }^{48}$ The choice of the GGA functional is motivated by the fact that for the substrate, GGA predicts slightly too large and the local-density approximation (LDA) slightly too low lattice constants, while for $\mathrm{Co}$ and Fe metals, the GGA predictions are definitely superior. In addition, the use of the GGA is crucial for the correct description of the structural and magnetic ground state of bulk ferromagnetic $3 d$ elements. ${ }^{49}$ The electron-ion interaction is described within the projectoraugmented-wave (PAW) (Ref. 50) formalism. The PAW potentials have been derived from fully relativistic calculations for atomic reference calculations. The PAW method reconstructs the full nodal character of the exact all-electron charge distribution in the core region. Hence it is a genuine all-electron approach that avoids the necessity to include nonlinear core corrections, which is of particular importance for magnetic systems. The basis set contained plane waves with a maximum kinetic energy of $300 \mathrm{eV}$, which allows to achieve full basis-set convergence.

Magnetic anisotropy and orbital moments are relativistic effects depending on the strength of the spin-orbit coupling. Spin-orbit coupling has been implemented in VASP by Kresse and Lebacq. ${ }^{51}$ Following Kleinman and Bylander ${ }^{26}$ and MacDonald et al. ${ }^{27}$ the relativistic Hamiltonian given in a basis of total angular momentum eigenstates $\left|j, m_{j}\right\rangle$ with $j=l \pm \frac{1}{2}$ (containing all relativistic corrections up to order $\alpha^{2}$, where $\alpha$ is the fine-structure constant) is recast in the form of 2 $\times 2$ matrices in spin space by reexpressing the eigenstates of the total angular momentum in terms of a tensor product of regular angular momentum eigenstates $|l, m\rangle$ and the eigenstates of the $z$ component of the Pauli-spin matrices. The relativistic effective potential consists of a term diagonal in spin space which contains the mass-velocity and Darwin corrections, and the spin-orbit operator. The nondiagonal elements in spin space arise from the spin-orbit coupling but also from the exchange-correlation potential when the system under consideration displays a noncollinear magnetization density. Calculations including spin-orbit coupling have therefore to be performed in the noncollinear mode implemented in VASP by Hobbs et al. ${ }^{52}$ and Marsman and Hafner. ${ }^{53}$

The substrate has been modeled by periodically repeated slabs consisting of up to ten atomic layers separated by a sufficiently thick vacuum space of $\simeq 16 \AA$. A $5 \times 5$ surface unit cell has been used with one adatom per surface cell. This corresponds to a coverage of $0.04 \mathrm{ML}$, only slightly larger than in experiment. Calculations on larger surface cells (and hence lower coverages) would require a much increased computational effort. In earlier experiments ${ }^{12}$ on $\mathrm{Co} / \mathrm{Pt}(111)$ for coverages varying between 0.007 and $0.03 \mathrm{ML}$, the MAE changed only from 9.2 to $8.6 \mathrm{meV} /$ atom. This indicates that our surface cell represents an acceptable compromise.

The adsorbates were placed on one side of the slab only and a dipole correction ${ }^{54,55}$ was applied to compensate for the electrical field gradient through the slab. Electronic and magnetic degrees of freedom were relaxed simultaneously until the change in total energy (TE) between successive iterations was smaller than $10^{-7} \mathrm{eV} /$ cell. Such a stringent relaxation criterion is essential for an accurate MAE. The coordinates of the adatom and the positions of the atoms in the three topmost layers of the substrate were optimized using scalar-relativistic calculations until the forces on all unconstrained atoms were converged to less than $0.01 \mathrm{eV} / \AA$. The geometry and electronic and magnetic ground state resulting from the scalar-relativistic calculation were used to initialize relativistic calculations including (SOC). No additional relaxation of the geometry was performed because our earlier work on $\mathrm{Fe}$ and $\mathrm{Co}$ adatom on $\mathrm{Pt}(111)$ has demonstrated that relaxations with and without SOC lead to almost identical results. ${ }^{30}$ Our description of SOC allows, in principle, for a noncollinear orientation of spin and orbital moments but we always found a collinear alignment. Two sets of selfconsistent calculations were performed for magnetic moments oriented perpendicular and parallel to the surface to find the easy and hard magnetic axes and to determine the MAE in terms of the difference in the total energies.

In parallel, the MAE was also calculated using the magnetic force theorem from the difference in the band energies 
at a frozen potential. The results achieved from the force theorem are, in general, less accurate but using the layer and angular momentum decomposed partial local densities of states, the MAE may be decomposed into contributions from the adatom and from different layers of the substrate, as well as into contributions from different orbitals. However, the calculation of the partial local densities of states must be based on the projection of the plane-wave components of the electronic eigenstates onto spherical waves within atomic spheres. It must be kept in mind that this approximate decomposition introduces additional numerical inaccuracies. However, this decomposition is very helpful in elucidating the electronic origin of the MAE.

The calculations including SOC require a fine $k$-point mesh for the Brillouin-zone integrations. Test calculations were performed for a Co adatom on a $\mathrm{Rh}(111)$ surface for three different $k$-point grids: $3 \times 3 \times 1,3 \times 3 \times 2$, and $5 \times 5$ $\times 1$ generated by the Monkhorst-Pack scheme ${ }^{56}$ and a modest Gaussian smearing of the eigenstates. A $3 \times 3 \times 1$ grid was found to provide a reasonable compromise between accuracy and computational effort. All converged total energies were extrapolated to zero smearing.

We should point out that our setup differs considerably from that used in previous calculations $s^{4,31,32,34}$ of the magnetic anisotropy of supported nanostructures. Most of these calculations are based on a KKR Green's-function approach, a local exchange-correlation functional, a complete neglect of relaxation of the adsorbate/substrate complex, and the use of the magnetic force theorem for calculating the magnetic anisotropy energy. A detailed comparative study of the impact of these approximations on calculations of the magnetic anisotropy energy has recently been published for $\mathrm{Fe}$ and $\mathrm{Co}$ adatoms on a $\mathrm{Pt}(111)$ surface. ${ }^{30}$

\section{COMPUTATIONAL RESULTS}

\section{A. Bulk metals and pristine surfaces}

The lattice constants of fcc Pd and Rh calculated using the GGA are (experimental values are given in brackets) $a_{\mathrm{Pd}}$ $=3.957(3.891) \AA$ (Ref. 57) and $a_{\mathrm{Rh}}=3.825(3.803) \AA, 58,59$ and the bulk moduli are $B_{\mathrm{Pd}}=174(180) \mathrm{GPa}$ (Ref. 57) and $B_{\mathrm{Rh}}=262(269) \mathrm{GPa} .{ }^{58,59}$ These lattice parameters have been applied in all further calculations. For Pd, we performed a comparative calculation using a local LDA functional, with the results $a_{\mathrm{Pd}}=3.866 \AA$ and $B_{\mathrm{Pd}}=210 \mathrm{GPa}$.

For a clean $\operatorname{Pd}(111)$ surface, the distances between the three top layers have been relaxed, We find interlayer relaxations of $\Delta_{12}=+0.2(0.3) \%$ and $\Delta_{23}=-0.5(-0.4) \%$ in calculations using five(ten)-layer slabs, to be compared with the available low-energy electron diffraction data finding an expansion of the first interlayer spacing by about $+1 \% .^{60-62}$ For the work function, we find $\Phi=5.30(5.32) \mathrm{eV}$ while experiment yields $\Phi=5.60 \mathrm{eV} .{ }^{63}$ At this level, one is led to conclude that a five-layer slab describes the structure of a $\operatorname{Pd}(111)$ surface with good accuracy. A problem arises, however, from the high polarizability of the Pd atoms. A spinpolarized GGA calculation for the five-layer slab leads to a weakly magnetic slab, the largest moments of $0.4 \mu_{B}$ are calculated for the central layer. In a calculation with a ten-layer slab, this spurious surface-induced magnetism disappears, all moments drop below $0.005 \mu_{B}$ even if the initialization assumes a magnetic moment. The results are essentially independent of the choice of the functional, spin-polarized LDA calculations lead to almost identical results for the geometry, the maximal moment in a five-layer slab is $0.3 \mu_{B}$. Hence it seems to be indicated to use a ten-layer slab in all calculations-however, this statement has to be revised when a magnetic atom is adsorbed at the surface.

The results for a $\mathrm{Rh}(111)$ surface are very similar. The surface-induced relaxations are $\Delta_{12}=-1.2 /-1.1 /-0.9 \%$ and $\Delta_{23}=0.6 / 0.0 / 0.0 \%$ for a five-layer, an eight-layer slab and in experiment. ${ }^{64}$ The work function is $\Phi=5.25 / 5.22 / 5.30 \mathrm{eV}$ (experiments by Brault et $a{ }^{65}{ }^{65}$ ). The spurious surfaceinduced moments are smaller already for the five-layer slab (maximal moment $0.11 \mu_{B}$ ), an eight-layer slab is nonmagnetic. However, the magnetism induced by an adatom or adlayer tends to be very long ranged and deserves attention.

\section{B. Geometry of the adsorption complex}

Table I summarizes the binding energies and the geometric data for isolated $\mathrm{Fe}$ and $\mathrm{Co}$ atoms adsorbed in threefold hollows and in twofold bridge positions on $\mathrm{Pd}$ and $\mathrm{Rh}$ (111) surfaces as calculated in a spin-polarized scalar-relativistic approach. Both fcc and hcp hollows have been considered and the binding energy at the bridge site has been calculated in order to provide an estimate for the diffusion barrier. The most important results are the following: On a Pd(111) surface, both Fe and Co adatoms prefer to occupy an hcp hollow, the difference in the binding energies is $39 \mathrm{meV} / \mathrm{atom}$ and $36 \mathrm{meV} /$ atom, respectively. The diffusion barrier for $\mathrm{Fe}(\mathrm{Co}) / \mathrm{Pd}(111)$ given by the difference in the binding energies at bridge and the more stable hcp hollow sites is $E_{\text {diff }}$ $\approx 144(128) \mathrm{meV}$. Using a typical value for the attempt frequency of $\nu_{0}=1 \times 10^{13} \mathrm{~Hz}$, we derive onset temperatures where the jump rate is $\nu=1 \mathrm{~Hz}$ of $T=41(36) \mathrm{K}$ for the relaxation from fcc to hep sites and of $T=56(50) \mathrm{K}$ for surface diffusion, respectively. Thus thermal diffusion is prohibited at the substrate temperatures used for atom deposition and for recording of the XMCD spectra. It is intuitive to consider nonthermal motion over a few lattice sites along the surface possibly caused by the dissipation of the adsorption energy. However, this so-called transient mobility has been precluded for all metal/metal systems where direct atomic-scale investigations exist. ${ }^{66}$ One example is $\mathrm{Pt} / \mathrm{Pt}(111)$, for which field ion microscopy has reported an equal occupation of fcc and hcp sites at $20 \mathrm{~K}$, despite the fcc site being more stable, as evidenced by diffusion from hcp to fcc hollows upon raising the temperature to $45 \mathrm{~K} .{ }^{67}$ Similarly for Co/Pt(111) STM has reported adsorption on both hollow sites with equal probability. ${ }^{15}$ Based on present knowledge, metal atoms deposited at low temperature onto a metal substrate therefore come to rest at their impact site implying instantaneous dissipation of the adsorption energy of several electron volts. We conclude that $\mathrm{Fe}$ and $\mathrm{Co}$ atoms investigated in experiment are with equal probability on hep and fcc sites on $\operatorname{Pd}(111)$. In both locations, they relax strongly inward by $0.63 \AA(\mathrm{Fe})$ and $0.66 \AA(\mathrm{Co})$, corresponding to $28 \%$ and 
TABLE I. The calculated adsorption energy $E_{\text {ad }}$, work function change $\Delta \Phi$ (with respect to the clean surface), height $z$ of the adatom above the surface, distance between adatom and the nearest surface atom $d$, relaxation $\Delta_{i j}$ of the interlayer distances, and the buckling of the substrate, $b_{i}$. The calculations are performed for five-layer thick slabs and the values quoted in brackets are obtained for a ten- and eight-layer slab for Pd and Rh substrates, respectively.

\begin{tabular}{|c|c|c|c|c|c|c|c|c|}
\hline Adsorption site & $\begin{array}{c}E_{\mathrm{ad}} \\
(\mathrm{eV})\end{array}$ & $\begin{array}{c}\Delta \Phi \\
(\mathrm{eV})\end{array}$ & $\begin{array}{c}z \\
(\AA)\end{array}$ & $\begin{array}{c}d \\
(\AA)\end{array}$ & $\begin{array}{l}\Delta_{12} \\
(\%)\end{array}$ & $\begin{array}{l}\Delta_{23} \\
(\%)\end{array}$ & $\begin{array}{c}b_{1} \\
(\AA)\end{array}$ & $\begin{array}{c}b_{2} \\
(\AA)\end{array}$ \\
\hline \multicolumn{9}{|c|}{$\mathrm{Fe} / \mathrm{Pd}(111)$} \\
\hline hcp hollow & 4.006 & -0.36 & 1.67 & 2.37 & +0.4 & -0.3 & 0.06 & 0.07 \\
\hline (hcp hollow) & 4.123 & $(-0.33)$ & (1.64) & $(2.36)$ & $(+0.6)$ & $(-0.1)$ & $(0.04)$ & $(0.07)$ \\
\hline fcc hollow & 3.967 & -0.36 & 1.67 & 2.37 & +0.4 & -0.3 & 0.07 & 0.04 \\
\hline bridge & 3.862 & -0.33 & 1.61 & 2.32 & +0.7 & -0.1 & 0.22 & 0.06 \\
\hline \multicolumn{9}{|c|}{$\mathrm{Co} / \mathrm{Pd}(111)$} \\
\hline hep hollow & 3.963 & -0.29 & 1.64 & 2.62 & +0.3 & -0.4 & 0.04 & 0.07 \\
\hline fcc hollow & 3.927 & -0.30 & 1.66 & 2.63 & +0.4 & +0.4 & 0.06 & 0.04 \\
\hline bridge & 3.835 & -0.28 & 1.62 & 2.66 & +0.4 & -0.4 & 0.21 & 0.08 \\
\hline \multicolumn{9}{|c|}{$\mathrm{Fe} / \mathrm{Rh}(111)$} \\
\hline hcp hollow & 5.180 & -0.34 & 1.76 & 2.38 & -0.7 & +0.7 & 0.04 & 0.02 \\
\hline (hcp hollow) & 5.289 & $(-0.37)$ & $(1.76)$ & $(2.38)$ & $(-0.9)$ & $(+0.1)$ & $(0.05)$ & $(0.02)$ \\
\hline fcc hollow & 5.091 & -0.36 & 1.81 & 2.39 & -0.6 & +0.7 & 0.06 & 0.01 \\
\hline bridge & 4.982 & -0.36 & 1.80 & 2.32 & -0.7 & +0.7 & 0.07 & 0.03 \\
\hline \multicolumn{9}{|c|}{$\mathrm{Co} / \mathrm{Rh}(111)$} \\
\hline hcp hollow & 5.333 & -0.28 & 1.73 & 2.36 & -0.7 & +0.7 & 0.05 & 0.03 \\
\hline (hcp hollow) & 5.438 & $(-0.28)$ & $(1.72)$ & $(2.36)$ & $(-0.9)$ & $(+0.1)$ & $(0.05)$ & $(0.01)$ \\
\hline fcc hollow & 5.245 & -0.29 & 1.76 & 2.37 & -0.6 & +0.7 & 0.07 & 0.02 \\
\hline bridge & 5.114 & -0.31 & 1.78 & 2.30 & -0.7 & +0.7 & 0.06 & 0.03 \\
\hline
\end{tabular}

$29 \%$ of the ideal interlayer distance. The interlayer distances in the substrate are hardly affected but the adsorption of the adatom causes also a weak buckling in the two top layers, the substrate atoms are attracted toward the adatom. The adsorption induces a decrease in the work function. Calculations based on a ten-layer slab are shown only for Fe(hcp)/ $\operatorname{Pd}(111)$ - except for a slightly increased binding energy, the increased slab thickness has no significant effect on the adsorption geometry.

On the $\mathrm{Rh}(111)$ surface the preference for hcp adsorption sites is even more pronounced, the differences in the binding energies are nearly identical, $89 \mathrm{meV}$ for $\mathrm{Fe}$ and $88 \mathrm{meV}$ for $\mathrm{Co}$, respectively. The diffusion barrier for $\mathrm{Fe}(\mathrm{Co})$ atoms is 198 (219) $\mathrm{meV}$, which is only slightly larger than twice the difference between the adsorption energies in the two hollows. The onset temperatures for relaxation from the metastable to the stable hollow are $T=42(51) \mathrm{K}$ and the ones for surface diffusion $T=77(85) \mathrm{K}$. Again, from comparison of these temperatures with the temperature used in experiment, and from the absence of transient mobility, the atoms are expected to be with equal probability on fcc and hcp sites. The inward relaxation of the adatoms is slightly smaller than on the Pd surface, $21 \%$ for $\mathrm{Fe}$ and $22 \%$ for $\mathrm{Co}$, as expected because of the smaller lattice constant of Rh. In an hcp hollow, the adatom moves closer to the surface than in a fcc site. The adsorption of an adatom also reduces the outward relaxation of the top layer of the substrate, the buckling of the surface at the adatom is weaker than on $\operatorname{Pd}(111)$.

\section{Magnetic properties}

Like magnetic impurities in highly polarizable hosts, $\mathrm{Fe}$ and Co adatoms on Pd and Rh surfaces induce a large polarization cloud in the substrate. Together, the moment of the adatom and the induced magnetization yield a very large total moment per magnetic atom. As shown in previous studies of the magnetic doping of transition-metal surfaces, the magnetic impurities induce a long-range oscillatory decaying polarization in the substrate ${ }^{37}$ In Table II, the spin moments of the adatom, the induced moments on the nearest neighbors (NNs) in the substrate, the total induced moments (integrated over all substrate atoms), and the resulting total spin moment per adatom for scalar-relativistic calculations (not including spin-orbit coupling) are compiled. The analysis of these data leads to several interesting conclusions: (i) although Fe adatoms have a higher magnetic moment than Co atoms, the magnetic moments induced by a Co adatom in a $\mathrm{Rh}(111)$ substrate are much larger. The reason is that due to the larger exchange splitting of the $\mathrm{Fe} d$ band, the overlap with the $\mathrm{Rh} d$ band is reduced, leading to a weaker hybridization and further to a reduced induced moment. On a $\operatorname{Pd}(111)$ substrate, the difference is less pronounced because as a consequence of the larger filling of the $\operatorname{Pd} d$ band the adatom/ substrate hybridization is reduced for both adatoms. (ii) On both substrates and for both adsorption geometries, the largest induced moments are always found on the three substrate atoms directly binding to the adatom but the moments 
TABLE II. Magnetic spin moments (in $\mu_{B}$ ) of adatoms, induced spin moments on nearest (NN) and next-nearest (NNN) neighbor atoms in top layer (upper line) and subsurface layer (second line) of the substrate, total induced spin moment of the substrate, and total spin moments for isolated $\mathrm{Fe}$ and $\mathrm{Co}$ atoms on $\mathrm{Rh}(111)$ and $\mathrm{Pd}(111)$, as resulting from scalar-relativistic calculations. The results have been obtained for a five-layer slab, numbers in parentheses have been calculated for a ten- (Pd) and an eight-layer (Rh) slab, respectively.

\begin{tabular}{|c|c|c|c|c|c|}
\hline & $\mu_{S}(\mathrm{Fe} / \mathrm{Co})$ & $\mu_{S}(\mathrm{Pd})_{\mathrm{NN}}$ & $\mu_{S}(\mathrm{Pd})_{\mathrm{NNN}}$ & $\mu_{S}(\mathrm{Pd})$ & $\mu_{S}(\mathrm{Fe} / \mathrm{Co}+\mathrm{Pd})$ \\
\hline \multirow[t]{2}{*}{$\mathrm{Fe}(\mathrm{hcp}) / \mathrm{Pd}(111)$} & \multirow[t]{2}{*}{$3.39(3.39)$} & $0.33(0.32)$ & $0.07(0.04)$ & \multirow[t]{2}{*}{$0.60(-0.53)$} & \multirow[t]{2}{*}{$3.99(2.86)$} \\
\hline & & $0.15(0.07)$ & $0.10(0.03)$ & & \\
\hline \multirow[t]{2}{*}{$\mathrm{Fe}(\mathrm{fcc}) / \mathrm{Pd}(111)$} & \multirow[t]{2}{*}{3.38} & 0.32 & 0.07 & \multirow[t]{2}{*}{0.81} & \multirow[t]{2}{*}{4.19} \\
\hline & & 0.11 & 0.04 & & \\
\hline \multirow[t]{2}{*}{$\mathrm{Co}(\mathrm{hcp}) / \mathrm{Pd}(111)$} & \multirow[t]{2}{*}{2.25} & 0.35 & 0.09 & \multirow[t]{2}{*}{0.25} & \multirow[t]{2}{*}{2.50} \\
\hline & & 0.17 & 0.13 & & \\
\hline \multirow[t]{3}{*}{$\mathrm{Co}(\mathrm{fcc}) / \mathrm{Pd}(111)$} & \multirow[t]{2}{*}{2.23} & 0.33 & 0.08 & \multirow[t]{2}{*}{0.62} & \multirow[t]{2}{*}{2.85} \\
\hline & & 0.15 & 0.06 & & \\
\hline & $\mu_{S}(\mathrm{Fe} / \mathrm{Co})$ & $\mu_{S}(\mathrm{Rh})_{\mathrm{NN}}$ & $\mu_{S}(\mathrm{Rh})_{\mathrm{NNN}}$ & $\mu_{S}^{\mathrm{tot}}(\mathrm{Rh})$ & $\mu_{S}(\mathrm{Fe} / \mathrm{Co}+\mathrm{Rh})$ \\
\hline \multirow[t]{2}{*}{$\mathrm{Fe}(\mathrm{hcp}) / \mathrm{Rh}(111)$} & \multirow[t]{2}{*}{$3.22(3.20)$} & $0.27(0.26)$ & $0.01(0.01)$ & \multirow[t]{2}{*}{$1.58(0.19)$} & \multirow[t]{2}{*}{$4.80(3.40)$} \\
\hline & & $0.05(0.04)$ & $-0.01(-0.01)$ & & \\
\hline \multirow[t]{2}{*}{$\mathrm{Fe}(\mathrm{fcc}) / \mathrm{Rh}(111)$} & \multirow[t]{2}{*}{3.21} & 0.26 & 0.04 & \multirow[t]{2}{*}{2.34} & \multirow[t]{2}{*}{5.05} \\
\hline & & 0.01 & -0.01 & & \\
\hline \multirow[t]{2}{*}{$\operatorname{Co}(\mathrm{hcp}) / \mathrm{Rh}(111)$} & \multirow[t]{2}{*}{$2.10(2.07)$} & $0.41(0.38)$ & $0.09(0.08)$ & \multirow[t]{2}{*}{$5.70(0.81)$} & \multirow[t]{2}{*}{$7.80(2.89)$} \\
\hline & & $0.07(0.08)$ & $0.02(0.02)$ & & \\
\hline \multirow[t]{2}{*}{$\mathrm{Co}(\mathrm{fcc}) / \mathrm{Rh}(111)$} & \multirow[t]{2}{*}{2.06} & 0.38 & 0.10 & \multirow[t]{2}{*}{5.39} & \multirow[t]{2}{*}{7.45} \\
\hline & & 0.03 & 0.01 & & \\
\hline
\end{tabular}

induced on the more distant atoms depend on substrate and adsorption site. For an adatom in an fcc hollow on $\mathrm{Rh}(111)$, the second largest induced moments are found on nextnearest-neighbor (NNN) sites in the surfaces plane while the moments induced in the subsurface layer are much smaller-in contrast to adatoms in an hep hollow which induce significant moments also in the subsurface layer. The difference is even more pronounced on $\operatorname{Pd}(111)$ where the second largest moment is found on the Pd atom in the second layer, directly below the adatom. This indicates that the adatom/substrate hybridization involves $d$ states with different symmetry, depending on the adsorption site. (iii) While the moments induced on nearest-neighbor sites are almost independent of the slab thickness, the integrated spin moments decrease strongly for thicker slabs. This is the consequence of the slow oscillatory decay of the induced magnetization with the distance from the magnetic impurity, as discussed in detail by Dennler et al. ${ }^{37}$ for magnetically doped Rh surfaces.

These results highlight one of the main difficulties of all density-functional studies of magnetic nanostructures supported on highly polarizable substrates. If the calculations are based on a slab which is sufficiently thick to produce a nonmagnetic ground state in the absence of any magnetic impurity, the integrated total induced moment depends very strongly on the thickness of the slab, in particular, we note a decrease in the induced and effective moments if the thickness of the slab is increased. This surprising result is due to the fact that at the lower surface of the slab the small induced negative moment is enhanced by the reduced coordination at the surface and the overestimation of the magnetic polarizability characteristic for the DFT. The effect is further enhanced because the very small induced moments from many atoms add up to a significant contribution. The conclusion is that a tremendous computational effort would be needed to bring the results on the magnetic properties of the adatom/ substrate complex to convergence with respect to the thickness of the slab. For this reason we shall confine our calculations of the magnetic anisotropy to slabs of five layers. At this slab thickness, the induced moments at the lower surface are nearly zero (a node in the oscillatory decaying moments falls close to the surface) and thus no artificially enhanced moment exists at the lower surface. Implicitly we make the assumption that the sum over the magnetic moments induced in the deeper layers is very small, although individual induced moments might be larger.

It might appear that investigations of such systems should be based on Green's-function methods for semi-infinite substrates rather than on a slab geometry. However, this conclusion would be premature. Our setup with a restricted slab thickness corresponds in fact to the usual practice in KKR Green's-function calculations where the Dyson equation is solved only for a small cluster surrounding the adatom. On all other substrate atoms, magnetism is suppressed by construction. Usually the number of atoms in such a cluster is much smaller than the number of atoms in the computational cell used for our five-layer slab. 
TABLE III. Spin $\mu_{S}$, orbital $\mu_{L}$, and total magnetic moments $\mu_{\text {tot }}$ (all in $\mu_{B}$ ) of Fe and Co adatoms adsorbed in fcc or hcp hollows on a $\mathrm{Rh}(111)$ substrate (five-layer slab) and induced moments on the Rh-substrate atoms integrated over all atoms in the supercell. Results obtained for perpendicular and in-plane orientations of the magnetic moments are presented. MAE (in millielectron volt) denotes the energy difference between the two orientations of the magnetic moments, calculated from the difference in the total energies (TEs) and using the magnetic force theorem (FT). A positive sign corresponds to a perpendicular easy axis. The energetically more stable adsorption site is marked by boldface.

\begin{tabular}{|c|c|c|c|c|c|c|c|c|c|c|}
\hline & \multicolumn{8}{|c|}{ Including SOC } & \multirow[b]{3}{*}{ MAE(TE) } & \multirow[b]{3}{*}{$\operatorname{MAE}(\mathrm{FT})$} \\
\hline & \multicolumn{4}{|c|}{ Perpendicular } & \multicolumn{4}{|c|}{ In plane } & & \\
\hline & $\mu_{S}$ & $\mu_{L}$ & $\mu_{L} / \mu_{S}$ & $\mu_{\mathrm{tot}}$ & $\mu_{S}$ & $\mu_{L}$ & $\mu_{L} / \mu_{S}$ & $\mu_{\mathrm{tot}}$ & & \\
\hline \multicolumn{11}{|c|}{ Fe in hcp hollow: } \\
\hline $\mathrm{Fe}$ & 3.216 & 0.078 & 0.024 & 3.294 & 3.216 & 0.087 & 0.027 & 3.303 & 0.07 & 0.02 \\
\hline $\mathrm{Rh}$ & 2.686 & 0.060 & 0.022 & 2.746 & 2.751 & 0.224 & 0.081 & 2.975 & & \\
\hline $\mathrm{Fe}+\mathrm{Rh}$ & 5.902 & 0.138 & 0.023 & 6.040 & 5.967 & 0.311 & 0.052 & 6.278 & & \\
\hline \multicolumn{11}{|c|}{$\mathrm{Fe}$ in fcc hollow: } \\
\hline $\mathrm{Fe}$ & 3.205 & 0.087 & 0.027 & 3.292 & 3.204 & 0.097 & 0.030 & 3.301 & -0.58 & -0.21 \\
\hline $\mathrm{Rh}$ & 2.617 & 0.057 & 0.021 & 2.674 & 2.835 & 0.238 & 0.084 & 3.073 & & \\
\hline $\mathrm{Fe}+\mathrm{Rh}$ & 5.822 & 0.144 & 0.025 & 5.966 & 6.039 & 0.335 & 0.055 & 6.374 & & \\
\hline \multicolumn{11}{|c|}{ Co in hep hollow: } \\
\hline Co & 2.094 & 0.141 & 0.067 & 2.235 & 2.095 & 0.147 & 0.070 & 2.242 & 1.65 & 0.16 \\
\hline $\mathrm{Rh}$ & 5.646 & 0.136 & 0.024 & 5.782 & 5.786 & 0.511 & 0.088 & 6.297 & & \\
\hline $\mathrm{Co}+\mathrm{Rh}$ & 7.740 & 0.277 & 0.036 & 8.017 & 7.881 & 0.658 & 0.083 & 8.539 & & \\
\hline \multicolumn{11}{|c|}{ Co in fec hollow: } \\
\hline $\mathrm{Co}$ & 2.061 & 0.158 & 0.076 & 2.219 & 2.060 & 0.166 & 0.081 & 2.226 & -0.29 & -0.14 \\
\hline $\mathrm{Rh}$ & 3.905 & 0.062 & 0.016 & 3.967 & 4.319 & 0.372 & 0.086 & 4.691 & & \\
\hline $\mathrm{Co}+\mathrm{Rh}$ & 5.966 & 0.220 & 0.037 & 6.186 & 6.379 & 0.538 & 0.084 & 6.917 & & \\
\hline
\end{tabular}

\section{Orbital moments and magnetic anisotropy}

Table III summarizes the results for spin and orbital moments and for the magnetic anisotropy energy of $\mathrm{Fe}$ and $\mathrm{Co}$ atoms supported on a $\mathrm{Rh}(111)$ surface. Both adsorption sites have been considered as they are expected to coexist in experiment.

Compared to the scalar-relativistic calculations (see Table II), the spin moment of the adatom remains almost unchanged but the total spin moment induced by an $\mathrm{Fe}$ adatom is strongly increased by SOC effects while for a Co adatom, the induced spin moment is decreased. The reason is that SOC leads to a mixing of different spin states, with the main contribution coming from states with a low excitation energy in the scalar-relativistic mode. Orbital moments on the adatom as well as the induced orbital moments remain smallmuch lower than the experimental estimates. The orbital moments are always parallel to the spin moment, as expected for late transition metals according to Hund's third rule.

While the spin moments of the adatoms are isotropic, we find a small anisotropy of the spin moments induced by $\mathrm{Fe}$ $\left(\Delta \mu_{S}=-0.065 /-0.261 \mu_{B}\right.$, a negative sign indicates that the moment is larger for in-plane orientation) and a much larger one for Co $\left(\Delta \mu_{S}=-0.140 /-0.414 \mu_{B}\right)$ adsorbed in an hcp/fcc hollow. In both cases, the induced spin moment is larger for in-plane orientation. A substantial anisotropy is found for the induced orbital moments, $\Delta \mu_{L}=-0.164 /-0.181 \mu_{B}$ for $\mathrm{Fe} /$ $\mathrm{Rh}(111)$ and $\Delta \mu_{L}=-0.375 /-0.310 \mu_{B}$ for $\mathrm{Co} / \mathrm{Rh}(111)$ in the hcp/fcc hollows. The orbital moments of the adatom are nearly isotropic.
For $\mathrm{Fe} / \mathrm{Rh}(111)$, we calculate from the change in the total energy a very small perpendicular magnetic anisotropy energy $(\mathrm{MAE}=0.07 \mathrm{meV})$ for an adatom in the energetically preferred hcp hollow while for the fcc hollow, we predict an in-plane easy axis (MAE $=-0.58 \mathrm{meV})$. For $\mathrm{Co}(\mathrm{hcp}) /$ $\mathrm{Rh}(111)$, we find a perpendicular anisotropy with MAE $=1.65 \mathrm{meV}$, for $\mathrm{Co}$ (fcc) an in-plane orientation of the magnetization is preferred, with a MAE of $-0.29 \mathrm{meV}$. The MAE has also been calculated using the force theorem. The results are in semiquantitative agreement with those derived from the total-energy differences, although — given the smallness of the MAE - they must certainly be considered as less reliable.

The site dependence of the magnetic anisotropy is a surprising result which has not been considered before (all earlier studies assumed that the adatom continues the bulk structure, with or without relaxation). The analysis of the anisotropies of the induced moments (which we find to be much stronger than that of the adatom) suggests that this might be a decisive factor determining the surprising change in the sign of the MAE. We find that the anisotropy of the induced spin moments is strongly site dependent-in contrast to the almost isotropic spin and orbital moments of the adatom $\left(\Delta \mu_{S(L)} \leq 0.01\right)$. The induced spin and orbital moments are always larger for in-plane orientation. For both $\mathrm{Fe}$ and $\mathrm{Co}$ in fcc hollows, we find a much larger induced inplane spin moment while for adatoms in an hep hollow, the spin anisotropy is reduced. The orbital anisotropy on the other hand is of comparable magnitude for both adsorption 
TABLE IV. Spin $\mu_{S}$, orbital $\mu_{L}$, and total magnetic moments $\mu_{\text {tot }}$ (all in $\mu_{B}$ ) of Fe and Co adatoms adsorbed on a Pd(111) substrate (five-layer slab) and induced moments on the Pd-substrate atoms integrated over all atoms in the supercell. Results obtained for perpendicular and in-plane orientations of the magnetic moment are presented. The MAE has been calculated as the difference in TEs and using the magnetic FT. A positive sign of the MAE (in millielectron volt) corresponds to a perpendicular easy axis.

\begin{tabular}{|c|c|c|c|c|c|c|c|c|c|c|}
\hline & \multicolumn{8}{|c|}{ Including SOC } & \multirow[b]{3}{*}{$\operatorname{MAE}(\mathrm{TE})$} & \multirow[b]{3}{*}{$\mathrm{MAE}(\mathrm{FT})$} \\
\hline & \multicolumn{4}{|c|}{ Perpendicular } & \multicolumn{4}{|c|}{ In plane } & & \\
\hline & $\mu_{S}$ & $\mu_{L}$ & $\mu_{L} / \mu_{S}$ & $\mu_{\text {tot }}$ & $\mu_{S}$ & $\mu_{L}$ & $\mu_{L} / \mu_{S}$ & $\mu_{\mathrm{tot}}$ & & \\
\hline \multicolumn{11}{|c|}{ Fe in hcp hollow: } \\
\hline $\mathrm{Fe}$ & 3.385 & 0.080 & 0.024 & 3.465 & 3.385 & 0.080 & 0.024 & 3.465 & -1.80 & -0.22 \\
\hline $\mathrm{Pd}$ & 0.639 & 0.028 & 0.044 & 0.667 & 0.395 & 0.019 & 0.048 & 0.414 & & \\
\hline $\mathrm{Fe}+\mathrm{Pd}$ & 4.024 & 0.108 & 0.027 & 4.132 & 3.780 & 0.099 & 0.026 & 3.879 & & \\
\hline \multicolumn{11}{|c|}{ Fe in fcc hollow: } \\
\hline $\mathrm{Fe}$ & 3.375 & 0.088 & 0.026 & 3.463 & 3.376 & 0.084 & 0.024 & 3.460 & 0.09 & 0.08 \\
\hline $\mathrm{Pd}$ & 0.866 & 0.054 & 0.062 & 0.920 & 0.886 & 0.085 & 0.096 & 0.971 & & \\
\hline $\mathrm{Fe}+\mathrm{Pd}$ & 4.241 & 0.142 & 0.033 & 4.383 & 4.262 & 0.169 & 0.040 & 4.431 & & \\
\hline \multicolumn{11}{|c|}{ Co in hep hollow: } \\
\hline Co & 2.245 & 0.220 & 0.098 & 2.465 & 2.244 & 0.188 & 0.083 & 2.432 & 1.61 & 0.72 \\
\hline $\mathrm{Pd}$ & -0.282 & -0.039 & 0.138 & -0.321 & -0.250 & -0.046 & 0.184 & -0.296 & & \\
\hline $\mathrm{Co}+\mathrm{Pd}$ & 1.963 & 0.181 & 0.092 & 2.144 & 1.994 & 0.142 & 0.071 & 2.136 & & \\
\hline \multicolumn{11}{|c|}{ Co in fec hollow: } \\
\hline Co & 2.236 & 0.247 & 0.110 & 2.483 & 2.244 & 0.218 & 0.097 & 2.462 & 2.27 & 1.88 \\
\hline $\mathrm{Pd}$ & 0.117 & 0.022 & 0.188 & 0.139 & 0.941 & 0.107 & 0.114 & 1.048 & & \\
\hline $\mathrm{Co}+\mathrm{Pd}$ & 2.353 & 0.269 & 0.114 & 2.622 & 3.185 & 0.325 & 0.102 & 3.510 & & \\
\hline
\end{tabular}

sites. The larger induced in-plane spin moments parallel the in-plane easy axis calculated for adatoms in the fcc hollows.

We have also investigated whether the results are affected in any way by increasing the slab thickness or the density of the $k$-point mesh. For an eight-layer slab of $\mathrm{Co}$ (fcc hollow)/ $\mathrm{Rh}(111)$, the preference for in-plane MAE is reduced from -0.29 to $-0.19 \mathrm{meV}$, for $\mathrm{Fe}($ hcp hollow)/Rh(111), the perpendicular MAE increases from 0.07 to $0.39 \mathrm{meV}$. Induced moments on nearest-neighbor sites are unchanged, although the total induced magnetization changes appreciably. This indicates that the oscillatory varying induced moments on the more distant sites have only a limited influence on the MAE, justifying the use of thinner slabs. This also suggests that the anisotropy of the total induced moments might not be of decisive importance for the strength of the MAE. For a five-layer slab of $\mathrm{Co} / \mathrm{Rh}(111)$, doubling the number of $k$ points changes the MAE only from -0.29 to $-0.25 \mathrm{meV}$. This demonstrates that these small energy differences are reasonably stable with respect to the computational setup.

Table IV summarizes the results for $\mathrm{Fe}$ and $\mathrm{Co}$ atoms on $\mathrm{Pd}(111)$. For a Co atom in an hcp/fcc hollow on $\operatorname{Pd}(111)$, we calculate orbital moments of $\mu_{L}=0.22(0.19) / 0.25(0.22) \mu_{B}$ for perpendicular (in-plane) magnetization, the spin moment is unchanged relative to the scalar-relativistic calculation and almost isotropic. At short distances from the adatom, induced spin and orbital moments are both parallel to the Co moment and almost independent of the direction of magnetization. The induced spin and orbital moments on the Pd atoms in the three top layers are shown in Fig. 1. At larger distances, the induced moments are aligned antiferromagnetically, leading to negative total induced spin and orbital moments and re- sulting in a modest total spin moment of $1.96(1.99) \mu_{B}$ at the hcp site. For a Co atom in an fcc hollow, the total induced spin and orbital moments remain positive, both are larger for
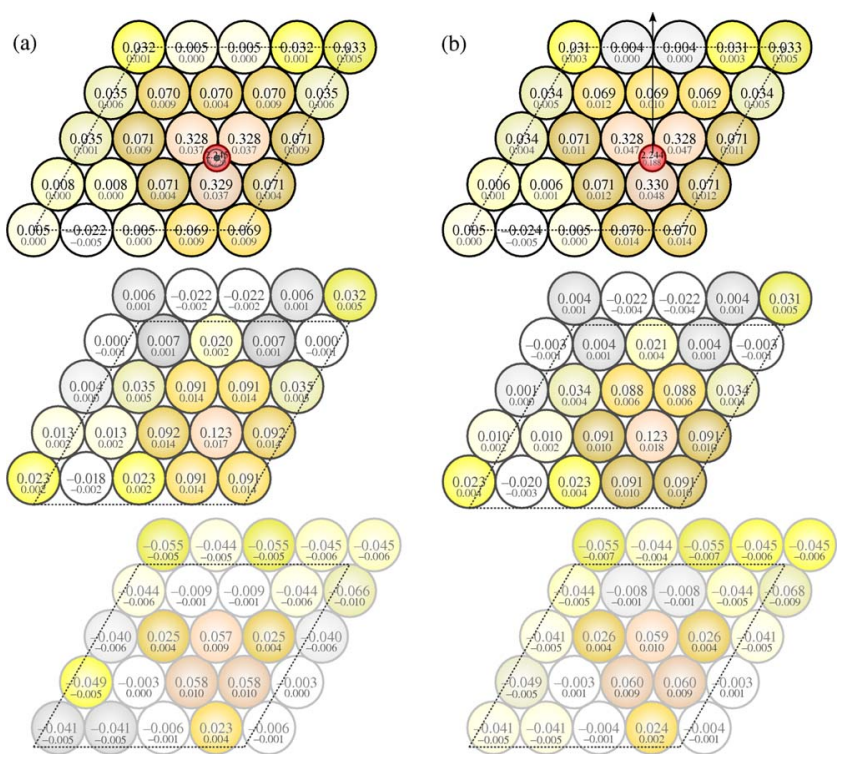

FIG. 1. (Color online) Magnetic spin (black) and orbital (gray) moments (in $\mu_{B}$ ) induced by an isolated Co adatom (adsorbed in an hep hollow on a five-layer Pd slab) on the Pd atoms in the first to third layer (top to bottom) with an (a) out-of-plane and (b) an inplane orientation of the moments as shown schematically by arrows. The shape of the surface unit cell applied in the calculations is also marked. 
in-plane magnetization. The anisotropy of both spin and orbital induced moments is larger for the fcc than for the hcp adsorption site but significantly lower than on the Rh substrate. We have to emphasize that the pronounced anisotropy of the induced moments is a rather long-range effect while the anisotropy of the moments induced on the nearestneighbor sites is much more modest (see Fig. 1). This statement has general validity for all systems investigated here.

For Co in both adsorption sites, the calculation predicts a perpendicular easy axis and a MAE of $1.61 \mathrm{meV}$ (hcp site) and $2.27 \mathrm{meV}$ (fcc site), respectively. The MAEs derived from the force theorem are also both positive but somewhat lower.

For an Fe atom in the energetically preferred hcp hollow, we calculate isotropic spin and orbital moments for the adatom, and a modest but strongly anisotropic induced spin moment and small induced orbital moments. Both spin and orbital induced moments are larger for perpendicular magnetization. The smallness of the induced moments arises again from a compensation between parallel and antiparallel induced moments. The magnetic easy axis lies in the plane with an MAE of $-1.80 \mathrm{meV}$. For an Fe atom in an fcc hollow, we find again almost isotropic spin and orbital moments (the spin moment is unchanged relative to the scalarrelativistic result) and-in contrast to an Fe atom in the hcp position-also almost isotropic induced spin and slightly anisotropic orbital moments.

The easy axis is perpendicular, the calculated MAE is $0.09 \mathrm{meV}$ (total-energy differences) and $0.08 \mathrm{meV}$ (force theorem). While the very low MAE for Fe in an fcc hollow corresponds to the low anisotropy of the moments of the adatom and of the ones induced in the substrate, the large negative MAE found for an adatom in an hcp hollow requires an explanation because only the induced spin moment displays a significant anisotropy, with the large value for out-of-plane magnetization.

\section{E. Electronic origin of the MAE}

Together with the previous study of $\mathrm{Fe}$ and Co adatoms on a $\mathrm{Pt}(111)$ substrate, ${ }^{30}$ the results described above allow to discuss some interesting trends. For a Co adatom, the hcp adsorption site is always preferred and we calculate an MAE of 1.19/1.61/1.65 meV on Pt, Pd, and Rh substrates. That the anisotropy energy on the $5 d$-substrate Pt should be lower than on the $4 d$-metals $\mathrm{Pd}$ and $\mathrm{Rh}$ with a lower spin-orbit coupling might at first sight be surprising but this is readily explained in terms of a reduced adatom/substrate hybridization reflected in a smaller overlap of the $d$ states of the adsorbate with the broader $d$ band of the $5 d$-metal substrate. The surprising result of the present calculations, however, is the strong predicted site dependence of the MAE: for a Co atom in the less-stable fcc hollow, on $\operatorname{Pd}(111)$ an enhanced positive MAE of $2.27 \mathrm{meV}$ is predicted while on $\mathrm{Rh}(111)$, we find even a change in sign of the MAE to $-0.29 \mathrm{eV}$. For an $\mathrm{Fe}$ adatom in fcc hollows, we calculate an MAE of 2.99/0.09/-0.58 meV for Pt, Pd, and Rh substrates. The fcc site is the preferred adsorption site only on Pt, and on Pd and $\mathrm{Rh}$ the sign of the MAE is different for both sites: 0.09/

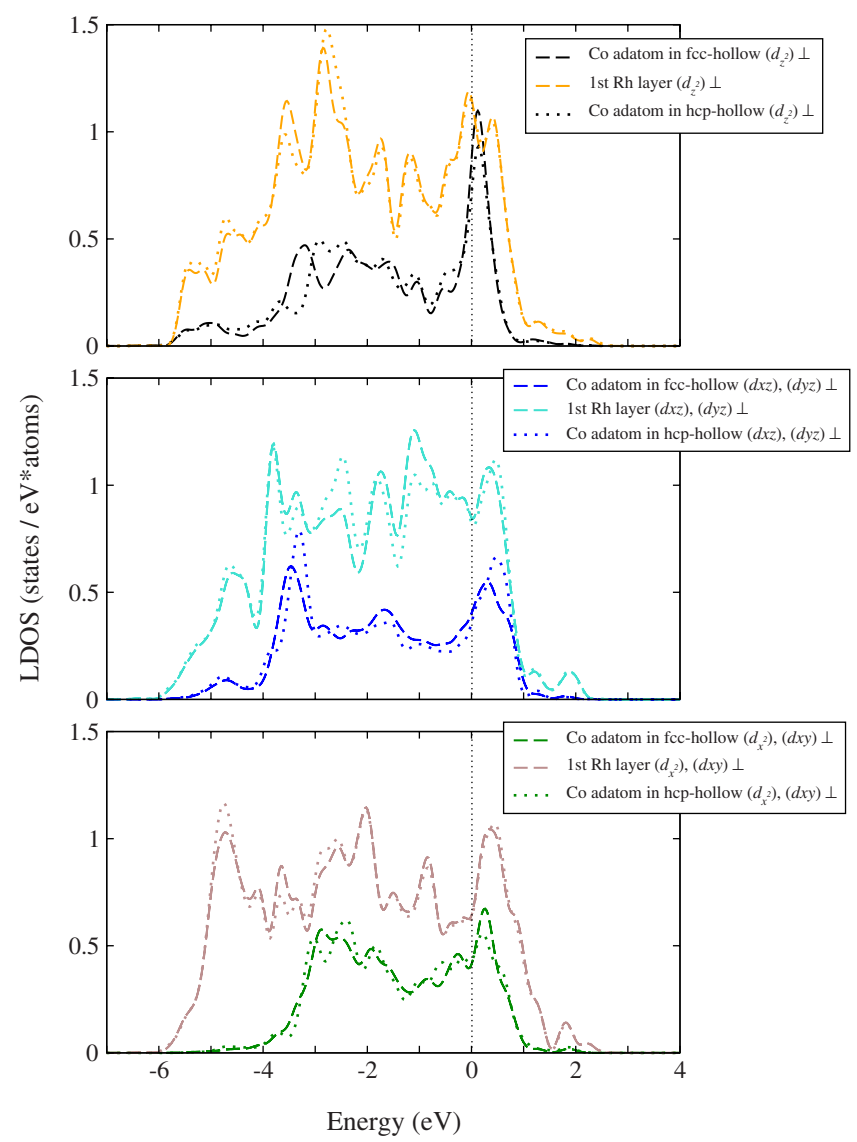

FIG. 2. (Color online) Partial electronic densities of states for a Co adatom on $\mathrm{Rh}(111)$ and for the $\mathrm{Rh}$ atoms in the surface layer, for the adatom in an fcc (dashed lines) or in an hcp (dotted lines) hollow. Cf. text.

$-1.80 \mathrm{meV}$ on $\mathrm{Pd}$ and $-0.58 / 0.07 \mathrm{meV}$ on $\mathrm{Rh}$ for the fcc/ hcp hollows. Hence we find that on Rh the adatom shows in-plane anisotropy in the fcc and out-of-plane anisotropy in the hcp hollows while on Pd, the trend is reversed. An Fe adatom has a perpendicular easy axis in the fcc, switching to in-plane for the hcp hollow. For the Co adatom, the easy axis remains perpendicular for both hollows but the MAE is reduced for the hep site.

The explanation of this surprising site dependence of the MAE must be sought in subtle differences in the hybridization between the $d$ states of the adatom and the substrate orbitals. Figure 2 shows the partial electronic densities of states (PDOSs) for the Co adatom and for the $\mathrm{Rh}$ atoms in the surface layer, for both adsorption sites. To simplify the picture and because in the presence of spin-orbit coupling, the spin is not a good quantum number and because the exchange splitting is very large for the adatom but only quite modest for the substrate, we prefer to plot the spinintegrated PDOS. Note that in all cases, the majority states of the adatom are completely filled so that the peak in the $\mathrm{Co}$ PDOS close to the Fermi level arises from minority states only. Due to the strong inward relaxation of the adatom, the distribution of the $d$ states of the magnetic adatom is strongly broadened by the hybridization with the substrate. The bandwidth of the Co $d$ states is a measure of the strength of this hybridization, it decreases in the sequence $d_{x z}, d_{y z} \rightarrow d_{z^{2}}$ 
$\rightarrow d_{x y}, d_{x^{2}-y^{2}}$. There are also subtle but significant differences between the PDOS for adatoms in the two hollows-the most important effects are found at the upper and lower band edges at the maxima associated with states that have predominantly spin-up and spin-down character. On the other hand, the changes associated with a reorientation of the magnetization direction are minimal-they hardly exceed the line thickness in these graphs. For the Co/Pd(111) system, the PDOS show a similar picture: the substrate bands are slightly narrower and due to the additional electron in the $\mathrm{Pd} d$ band the Fermi level falls close to the upper band edge. This also reduces the hybridization with the Co-minority $d$ states such that the corresponding PDOS peaks fall closer to the Fermi level.

The elucidation of the electronic origin of the MAE requires an even more detailed analysis, and here the decomposition of the MAE into adatom and substrate contributions, and into contributions from different angular momentum states permitted by the force theorem and calculated in terms of the band energies by integrating the PDOS is very helpful. Within this formalism, the MAE may be written as

$$
\mathrm{MAE}=\sum_{i} \sum_{m_{l}=-2}^{2} \int_{E_{B}}^{E_{F}}\left(E-E_{F}\right) \Delta n_{m_{l}}^{i}(E) d E,
$$

where the sum is over all atoms in the supercell and over all angular momentum quantum number $m_{l}, E_{B}$ is the energy at the bottom of the valence band, and where

$$
\Delta n_{m_{l}}^{i}(E)=n_{m_{l}}^{i}(E ; \perp)-n_{m_{l}}^{i}(E ; \|)
$$

is the difference in the partial local density of states for electrons with quantum number $m_{l}$ at the site $i$ for perpendicular $(\perp)$ and in-plane $(\|)$ magnetization. The orbital anisotropy $\Delta \mu_{L}=\mu_{L}^{\perp}-\mu_{L}^{\|}$may be calculated in terms of the difference in the occupation of states with $m_{l}= \pm 2, \pm 1$, i.e., according to

$$
\Delta \mu_{L}=2 \mu_{B} \sum_{i} \sum_{m_{l}=1,2} \int_{E_{B}}^{E_{F}}\left[\Delta n_{m_{l}}^{i}(E)-\Delta n_{m_{-l}}^{i}(E)\right] d E .
$$

Note that a large contribution to the MAE requires a large value of $\Delta n_{m_{l}}^{i}(E)$, integrated over the valence band, irrespective of the value of $m_{l}$. A significant orbital anisotropy arises only if $\Delta n_{m_{l}}^{i}(E)$ is different for states with $m_{l}= \pm 1$ and/or $m_{l}= \pm 2$. One must also remember that the expression given for the orbital anisotropy is exact whereas the force theorem leads only an approximate value for the MAE.

For hcp $\mathrm{Co} / \mathrm{Rh}(111)$, the contributions to the MAE are 0.19/0.12/0.06 meV for the Co adatom and the first and second Rh-substrate layers. This gives altogether an MAE of $0.37 \mathrm{meV}$, to be compared with $0.16 \mathrm{meV}$ calculated from the force theorem and $1.65 \mathrm{meV}$ from the self-consistent total-energy difference. For fcc $\mathrm{Co} / \mathrm{Rh}(111)$, the corresponding numbers are $0.06 /-0.13 /-0.08 \mathrm{meV}$ from $\mathrm{Co} / \mathrm{Rh} 1 / \mathrm{Rh} 2$, together $-0.15 \mathrm{meV}$ to be compared with a total MAE of $-0.14 \mathrm{meV}$ (FT) and $-0.29 \mathrm{meV}$ (TE). At this point, it must be emphasized that not only the force theorem is already an approximation. In addition, the decomposition of the DOS into its local and angular momentum components resides on an atomic-sphere projection and the individual PDOS are rather noisy. Hence the decomposition of the MAE has semiquantitative validity at best but it is still very helpful for the elucidation of the physical mechanisms.

The decomposition of the MAE shows that the negative sign for Co in the less-stable fcc hollows has a double origin: a reduced positive contribution from the Co adatom and a negative contribution from the substrate. Their origin can in turn be traced back to differences in the PDOS for perpendicular and in-plane orientation of the magnetization, shown in Fig. 3. The differences in the PDOS induced by a change in the direction of magnetization are very small, at most about $1 \%$ of the PDOS. The curves look rather noisy at first sight (even after a modest filtering) but the calculations show that the oscillations at higher binding energies largely cancel out and the dominant contributions come from states very close to the Fermi edge. Inspection of the differences in the PDOS shows for the Co adatom in the hep hollow positive contributions from the $d_{z}^{2}\left(m_{l}=0\right)$ and $d_{x z}, d_{y z}\left(m_{l}= \pm 1\right)$ states but in the fcc hollow small negative contributions from the $d_{z^{2}}$ and $d_{x^{2}-y^{2}}\left(m_{l}=2\right)$ and small positive contributions from the $d_{x z}, d_{y z}$ states. This analysis also explains the very small orbital anisotropy. A large $\Delta \mu_{L}$ would require that the degeneracy of the $d_{x z} / d_{y z}$ and $d_{x y} / d_{x^{2}-y^{2}}$ states for perpendicular magnetization is strongly broken for in-plane magnetization while we find that, especially close to the Fermi level these states remain almost degenerate (and hence do not contribute to the orbital anisotropy, while still contributing to the MAE). For the substrate contribution, a large positive contribution for $d_{z^{2}}$ and a smaller negative contribution from $d_{x z}, d_{y z}$ states is evident for Co in the hcp hollow. The increased occupation of the $m_{l}=0$ states also explains the smaller orbital moment for perpendicular magnetization (although the large value listed in Table III results from an integration over all substrate atoms). For Co in an fcc hollow, the differences in the PDOS of the Rh layer are small close to $E_{F}$, hence the negative contribution results also from states at higher binding energies. The important role of $d_{z^{2}}$ and $d_{x z}, d_{y z}$ states of both adatom and substrate in determining the site dependence of the MAE shows its close relation to small but significant modifications in the $d$ states extending perpendicular to the surface.

For $\mathrm{Co} / \mathrm{Pd}(111)$, we find a positive (perpendicular) MAE for both adsorption sites. For the adatom in the stable hcp hollow, we calculated contributions of $0.6 / 0.07 / 0.09 \mathrm{meV}$ for $\mathrm{Co} / \mathrm{Pd} 1 / \mathrm{Pd} 2$, i.e., together $0.76 \mathrm{meV}$, to be compared with an MAE of $0.72 \mathrm{meV}(\mathrm{FT})$ and $1.61 \mathrm{meV}$ (TE). For Co in an fcc hollow, the corresponding values are 1.11/0.22/0.34 meV from $\mathrm{Co} / \mathrm{Pd} 1 / \mathrm{Pd} 2$, total $1.67 \mathrm{meV}$, to be compared with an MAE of $1.88 \mathrm{meV}(\mathrm{FT})$ and $2.27 \mathrm{meV}$ (TE). In this case, the analysis of the differences in the PDOS shows almost the same picture for both adsorption sites. The dominant contributions to the MAE of both adatom and substrate come in both cases from an increased occupation of $d_{z^{2}}$ states, which is more pronounced for the fcc hollow.

For Fe adatoms the situation is even more complex. On both substrates, the calculations predict that the MAE changes sign between the two different adsorption sites. The larger spin moment of the $\mathrm{Fe}$ adatom is coupled to a larger exchange splitting and in turn to significant differences in the electronic spectrum. The PDOS for $\mathrm{Fe} / \mathrm{Pd}(111)$ is shown in 

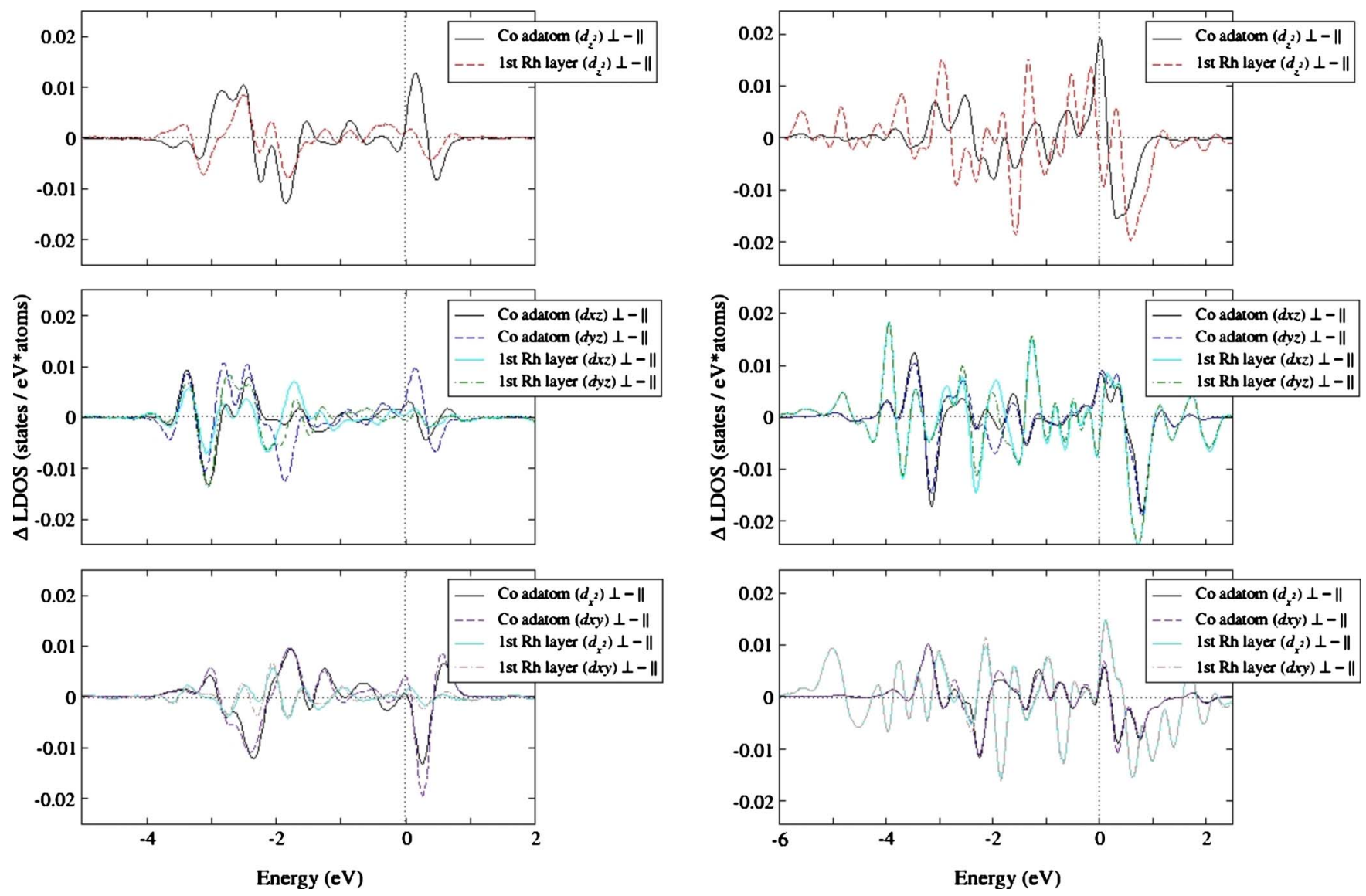

FIG. 3. (Color online) Differences in the partial electronic densities of states for a Co adatom on $\mathrm{Rh}(111)$ and for the Rh atoms in the surface layer as a function of the direction of magnetization, for the adatom in an fcc (left panels) and in an hcp (right panels) hollow. Cf. text.

Fig. 4. For all Fe $d$ states, the minority states form a sharp peak located about $0.5 \mathrm{eV}$ above the Fermi level. Hybridization of the states extending perpendicular to the surface with the substrate orbitals leads to the formation of a sharp resonance in the corresponding Pd states and the formation of a deep minimum in the Pd-PDOS at the Fermi level. Differences in the PDOS caused by a different location of the adatom are modest, the largest are found in the Fe $d_{z^{2}}$ majority states and in the $\mathrm{Pd} d_{x z}, d_{y z}$ states. For $\mathrm{Fe} / \mathrm{Rh}(111)$, the PDOS shows a similar picture but the Fermi level is downshifted due to the reduced $d$-band occupation in the substrate and the hybridization-induced PDOS minima are much less pronounced.

For hcp Fe/Pd(111), the decomposition of the MAE yields values of $-0.04 /-0.20 / 0.03 \mathrm{meV}$ for $\mathrm{Fe} / \mathrm{Pd} 1 / \mathrm{Pd} 2$, total $-0.21 \mathrm{meV}$, to be compared with an MAE of $-0.22 \mathrm{meV}$ (FT) and $-1.80 \mathrm{meV}$ (TE), for fcc Fe/Pd(111) we find $0.07 /$ $0.02 / 0.02 \mathrm{meV}$ for $\mathrm{Fe} / \mathrm{Pd} 1 / \mathrm{Pd} 2$, total $0.11 \mathrm{meV}$, to be compared with an MAE of $0.08 \mathrm{meV}$ (FT) and $0.09 \mathrm{meV}$ (TE). The decomposition shows that the in-plane anisotropy for $\mathrm{Fe}$ atoms in the hcp hollows stems from a small negative contribution of the adatom and a larger one from the first substrate layer. A detailed assignment of the origin of these contributions is more difficult than for Co adatoms. The differences in the PDOS as a function of the direction of the magnetization shown in Fig. 5 are very small at $E_{F}$ and for binding energies up to about $-1.5 \mathrm{eV}$. Hence according to the force theorem, the leading contributions to the MAE must be assigned to a change in the occupation of states located at larger binding energies.

For hcp Fe/Rh(111), the decomposition of the MAE yields contributions of $0.04 / 0.05 / 0.03 \mathrm{meV}$ for $\mathrm{Fe} / \mathrm{Rh} 1 / \mathrm{Rh} 2$, together $0.12 \mathrm{meV}$, in good agreement with the total MAE of $0.02 \mathrm{meV}$ (FT) or $0.07 \mathrm{meV}$ (TE). For fcc Fe/Rh(111), we find contributions of $0.12 /-0.10 /-0.02 \mathrm{meV}$ from $\mathrm{Fe} / \mathrm{Pd} 1 /$ $\mathrm{Pd} 2$ - in this case, the contributions balance exactly to zero whereas force theorem $(-0.21 \mathrm{meV})$ and total-energy differences $(-0.58 \mathrm{meV})$ yield a modest negative MAE. This shows that the negative MAE for Fe in the fcc hollow stems from the substrate contribution which overcompensates a positive MAE from the adatom. The analysis of the differences in the PDOS yields again a picture very similar to that described for $\mathrm{Fe} / \mathrm{Pd}(111)$. However, it must be admitted that for MAEs below $1 \mathrm{meV}$, we are certainly at the limit of the accuracy of DFT calculations and that the decomposition of the MAE according to the force theorem introduces additional approximations. The differences in the FT and TE values of the MAE should give rise to some caution against an overinterpretation of this kind of analysis. Still, the analysis of the PDOS leads to an important conclusion. Due to the large exchange splitting of the Fe $d$ states, the peak in the minority DOS is located above the Fermi energy, the Fe- 

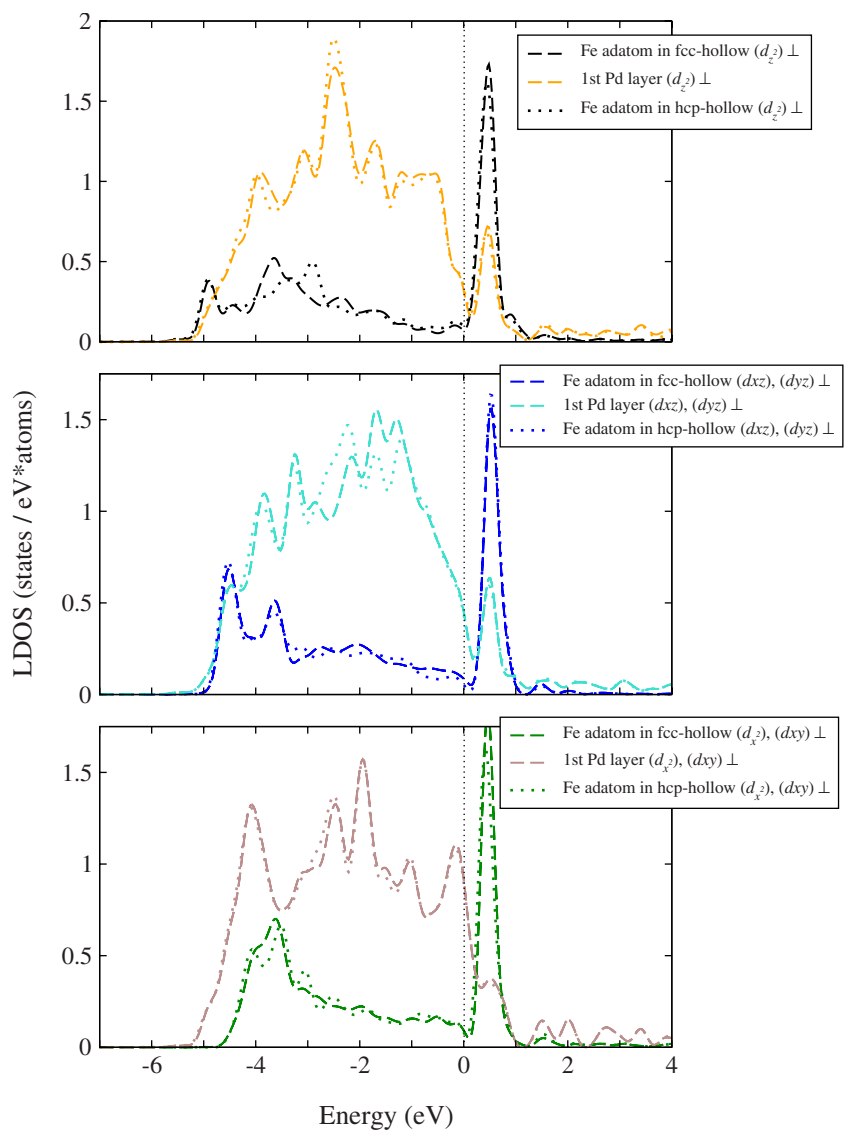

FIG. 4. (Color online) Partial electronic densities of states for an Fe adatom on $\mathrm{Pd}(111)$ and for the Pd atoms in the surface layer, for the adatom in an fcc (dashed lines) or an hcp (dotted lines) hollow. Cf. text.

DOS at $E_{F}$ is low and undergoes only very small variations as a function of the direction of magnetization. Contributions to the MAE stem from states with higher binding energies and they are small due to cancellation effects. This leads to the conclusion that Fe adatoms and, in general, Fe nanostructures should have a much smaller MAE than those formed by $\mathrm{Co}$ atoms, in spite of the much larger spin moment of Fe.

Our analysis also considerably modifies the picture of the origin of the MAE derived from earlier theoretical studies based on idealized nonrelaxed geometries. ${ }^{31,32,34}$ From LSDA calculations for $\mathrm{Fe}$ and $\mathrm{Co}$ adatoms on $\mathrm{Pt}(111)$ and $\operatorname{Ir}(111)$ substrates, Etz et al. ${ }^{34}$ concluded that even the $5 d$ substrates contribute only between $5.5 \%$ (for $\mathrm{Fe} / \mathrm{Pt}$ ) and $25 \%$ (for $\mathrm{Fe} / \mathrm{Ir}$ ) to the MAE and can in no case change the sign of the MAE determined by the adatom alone. Due to the weaker SOC, the contribution from a $4 d$ substrate could be expected to be even weaker. However, this result is due primarily to the neglect of relaxation which largely suppresses the hybridization between adatom and substrate states. For our fully relaxed adatom/substrate complexes, we find that even for the system with the by far largest MAE $[\mathrm{Co} / \mathrm{Pd}(111)]$, the substrate contributes about one third to the MAE and that as demonstrated for fcc $\mathrm{Co} / \mathrm{Rh}(111)$ the substrate contribution can even determine the sign of the MAE.

Another conclusion, already suggested by the comparison of orbital anisotropies and MAE, is that the popular assump- tion that the MAE is directly related to the orbital moment anisotropy $\Delta \mu_{L}$ such that the largest orbital moment is found along the easy magnetization direction favored by the MAE, is not generally valid. This assumption is based on the perturbation treatment of the MAE proposed by Bruno ${ }^{68}$ for materials with a single atomic species. In that case, perturbation theory predicts $\mathrm{MAE}=\frac{\xi}{4 \mu_{B}} \Delta \mu_{L}$, where $\xi$ stands for the strength of the SOC. It is evident that the relation between MAE and orbital anisotropy cannot be as simple as that if more than one atomic species is present and a strong hybridization exists between their electronic states. Andersson et $a l .{ }^{69}$ have shown that the MAE can be written as a sum over atomic species, $i$, and a double sum over occupied $(s)$ and unoccupied $\left(s^{\prime}\right)$ spin states, MAE $=\sum_{i s s^{\prime}} \Delta E_{i}^{s s^{\prime}}$, where the offdiagonal $\left(s \neq s^{\prime}\right)$ terms arise from the spin-orbit scattering at other sites. Even for $\mathrm{Au} / \mathrm{Co} / \mathrm{Au}$ trilayers where $\mathrm{Co}$ is coupled to a metal which has a strong SOC but only a very modest magnetic polarizability, Andersson et al. have demonstrated that the coupling to the ligands leads to strong deviations of the MAE from the linear correlation with $\Delta \mu_{L}$ expected on the basis of Brunos formula. For our systems, we find that only for $\mathrm{Co} / \mathrm{Pd}(111)$ at least the sign of the MAE is the same as that of the orbital anisotropy but the MAE is larger for Co in an fcc site while $\Delta \mu_{L}$ is larger for Co in an hep site. For the other systems, signs of MAE and orbital moment anisotropy agree as often as they disagree. The correlation is not improved if only local contributions to the MAE and localorbital moment anisotropies are compared. This emphasizes that, not unexpectedly, the off-site spin-orbit coupling plays a very important role. A further complication arises from the fact that although the spin moments of the adatoms are almost strictly isotropic, significant anisotropies are found for the induced spin moments. van der $\operatorname{Laan}^{70}$ has extended the perturbation approach to include spin moment anisotropy but again only for one-component systems.

\section{EXPERIMENT}

The magnetic properties of Fe adatoms on the (111) surfaces of $\mathrm{Pd}$ and $\mathrm{Rh}$, as well as the ones of Co adatoms on $\mathrm{Rh}(111)$, have been investigated in situ by means of XMCD at the ID08 beamline of the European Synchrotron Radiation Facility (ESRF) in Grenoble. Single crystal $\operatorname{Pd}(111)$ and $\mathrm{Rh}(111)$ substrates were prepared by $\mathrm{Ar}^{+}$-ion sputtering $\left(E_{\mathrm{kin}}=1.3 \mathrm{keV}\right)$ at $T=300 \mathrm{~K}$ followed by annealing at $T$ $=800 \mathrm{~K}$ at an oxygen partial pressure of $p_{\mathrm{O}_{2}}=6$ $\times 10^{-8} \mathrm{mbar}$ for $10 \mathrm{~min}$ to remove carbon impurities, and a final flash annealing to $1000-1100 \mathrm{~K}$. Substrate cleanliness was checked by Auger electron and X-ray absorption spectroscopy (XAS). Minute amounts of Fe and Co were deposited from high-purity rods $(99.995 \%)$ with an electron-beam evaporator on the substrate held at $T=8 \pm 1 \mathrm{~K}$. The deposition flux was $5 \times 10^{-4} \mathrm{ML} / \mathrm{s}$ and $1 \times 10^{-3} \mathrm{ML} / \mathrm{s}$ for Fe and Co, respectively.

As outlined in Sec. III B, thermal diffusion is inhibited at $8 \mathrm{~K}$ and transient mobility is absent for all investigated $\mathrm{metal} / \mathrm{metal}$ systems where direct atomic-scale information is available. Therefore, the $\mathrm{Fe}$ and $\mathrm{Co}$ atoms come to rest at their site of impact. This is referred to as statistical growth. 

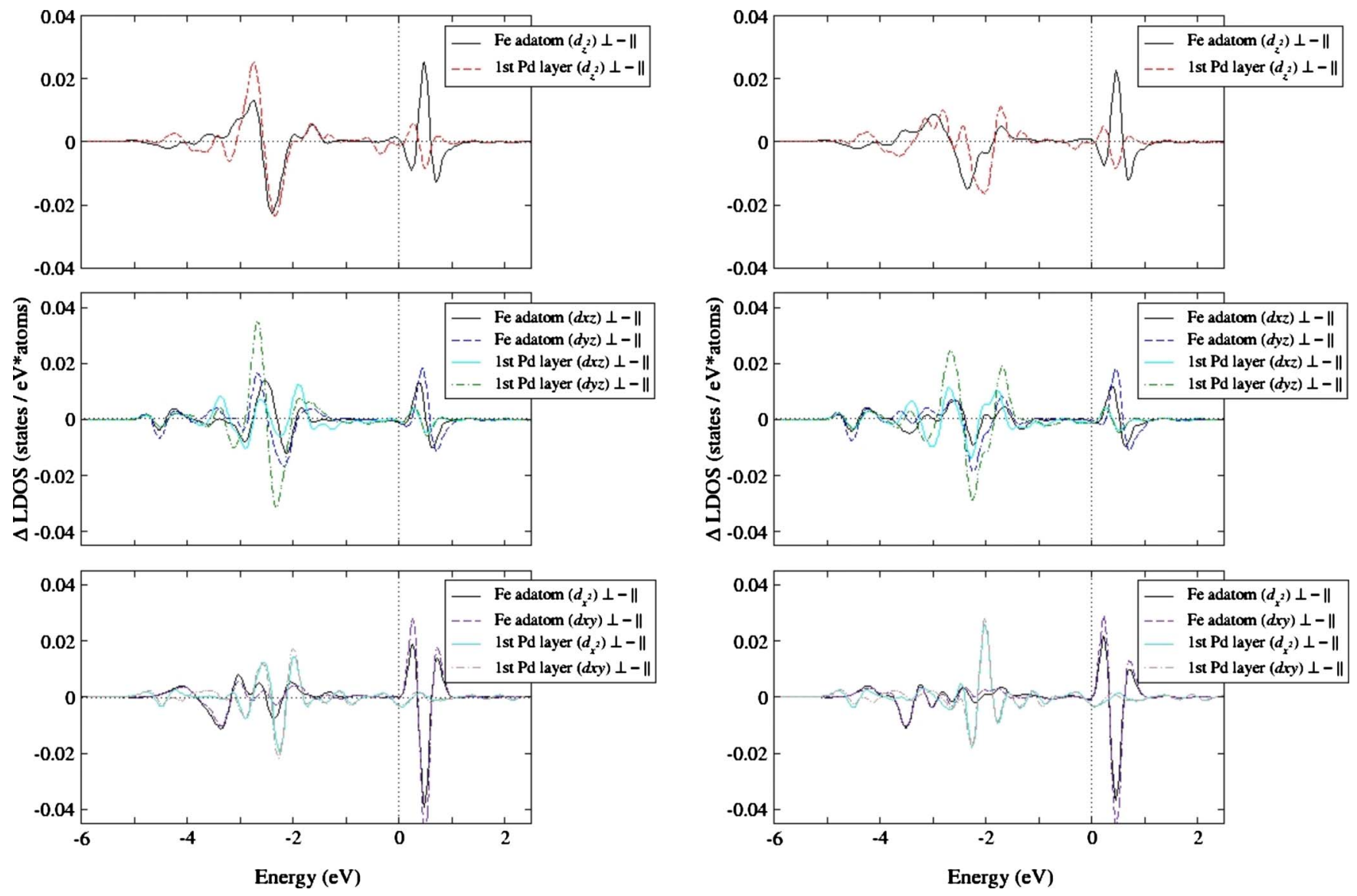

FIG. 5. (Color online) Differences in the partial electronic densities of states for an Fe adatom on Pd(111) and for the Pd atoms in the surface layer as a function of the direction of magnetization, for the adatom in an fcc (left panels) or an hcp (right panels) hollow. Cf. text.

For the coverages of $\Theta=0.01 \mathrm{ML}$ and $\Theta=0.02 \mathrm{ML}$ employed in this work, the probability of two adatoms landing on neighboring sites and thus forming a dimer is very low. The mean island sizes are 1.04 atoms at $0.01 \mathrm{ML}$ and 1.07 atoms at $0.02 \mathrm{ML} ; 97 \% / 3 \% / 0 \%$ and $93 \% / 6 \% / 1 \%$ of the islands are monomers/dimers/trimers for $0.01 \mathrm{ML}$ and 0.02 ML, respectively. ${ }^{8}$ We therefore probe essentially single atoms residing with equal probability in the two nonequivalent hollow sites.

Since the magnitude of the XMCD signal scales as the projection of the probed atomic moments onto the $\mathrm{x}$-ray incidence direction, out-of-plane vs in-plane magnetization measurements were carried out by rotating the sample position from $\theta=0^{\circ}$ to $\theta=70^{\circ}$, corresponding to normal and grazing incidence of the $\mathrm{x}$-ray beam, respectively. A magnetic field up to $5 \mathrm{~T}$ collinear with the $\mathrm{x}$-ray beam was applied. XAS experiments were performed at $T=8 \pm 1 \mathrm{~K}$ in the total electron yield (TEY) mode using $99 \pm 1 \%$ circularly polarized light.

Figure 6 shows the XAS and the resulting XMCD spectra for Fe on $\mathrm{Pd}(111)$ and on $\mathrm{Rh}(111)$ taken at the $\mathrm{Fe} L_{2,3}$ edges. The adatom XAS have relative weak absorption features compared with the Pd and Rh TEY background due to the small coverage of only $0.02 \mathrm{ML}$ and $0.01 \mathrm{ML}$, respectively. The small hump of the $L_{2}$ edge is hardly visible in the XAS but a clear $L_{2}$ peak can be detected in the XMCD signal. For $\mathrm{Fe}$ on $\mathrm{Pd}$ and on $\mathrm{Rh}$, the XMCD/XAS asymmetry is larger at
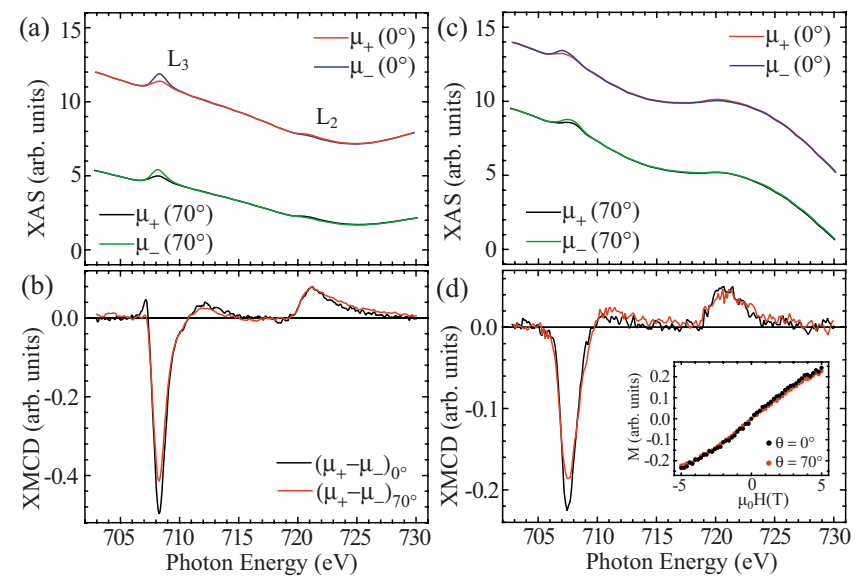

FIG. 6. (Color online) (a) and (b) $0.02 \mathrm{ML}$ Fe on Pd(111), (c) and (d) 0.01 ML Fe on Rh(111): (a) and (c) XAS and (b) and (d) XMCD spectra measured at the $\mathrm{Fe} L_{2,3}$ edges at $T=8 \mathrm{~K}$ and $B$ $=5 \mathrm{~T}$. In order to eliminate the dependence of the electron yield on the sample orientation, the XAS at $70^{\circ}$ and $0^{\circ}$ have been normalized to the background corrected area under the $L_{3}$ peak: $\int_{L_{3}}\left(\mu_{+}\right.$ $\left.+\mu_{-}\right)$. Inset in Fig. (d): magnetization curves at $\theta=0^{\circ}$ (black dots) and $70^{\circ}$ [red (gray) dots] measured by taking the peak height of the $L_{3} \mathrm{XMCD}$ intensity at $707.7 \mathrm{eV}$ divided by the pre-edge intensity at $705.0 \mathrm{eV}$ as a function of the applied magnetic field. 
TABLE V. Orbital to spin moment ratios estimated from XMCD spectra acquired along the easy magnetization axis at $B=5 \mathrm{~T}$ and MAE (millielectron volt per atom, positive values indicate out-ofplane easy axis).

\begin{tabular}{lccc}
\hline \hline & $\Theta(\mathrm{ML})$ & $\mu_{L} / \mu_{S+7 D}$ & MAE \\
\hline $\mathrm{Fe} / \mathrm{Pd}(111)$ & 0.02 & $0.12 \pm 0.05$ & $\geq 0$ \\
$\mathrm{Co} / \mathrm{Pd}(111)^{\mathrm{a}}$ & 0.02 & $0.70 \pm 0.06$ & $\geq 3$ \\
$\mathrm{Fe} / \mathrm{Rh}(111)$ & 0.01 & $0.15 \pm 0.05$ & $\geq 0$ \\
$\mathrm{Co} / \mathrm{Rh}(111)$ & 0.02 & $0.57 \pm 0.04$ & $-0.6 \pm 0.1$ \\
\hline \hline
\end{tabular}

Reference 73 .

$0^{\circ}$ suggesting an out-of-plane easy axis. The inset in Fig. 6(d) shows an example of field-dependent magnetization measured along the out-of-plane $\left(\theta=0^{\circ}\right)$ and close to inplane $\left(\theta=70^{\circ}\right)$ direction for $\mathrm{Fe} / \mathrm{Rh}(111)$. The $M(H)$ curves for Fe on $\mathrm{Pd}(111)$ are similar. At both angles, we observe two almost linear magnetization curves with similar slope, indicative of a very small MAE. Obviously, saturation is not reached and we cannot apply the XMCD sum rules ${ }^{71,72}$ to estimate the orbital and spin magnetic moment independently. However, quantitative information can be obtained by calculating the ratio $\mu_{L} / \mu_{S+7 D}$, where $\mu_{D}$ is the spin dipole moment. The obtained values along the easy axis are reported in Table V. We must caution, however, that both the magnitude ${ }^{74}$ and the anisotropy ${ }^{75}$ of $\mu_{7 D}$ can be substantial for magnetic impurities. These effects, related to the anisotropy of the spin density within the adatom unit cell, prevent a precise determination of the orbital to spin ratio and orbital moment anisotropy based on the comparison of $\mu_{L} / \mu_{S+7 D}$ values measured along different directions.

The XAS, XMCD spectra, and magnetization curves of $0.02 \mathrm{ML} \mathrm{Co} / \mathrm{Rh}(111)$ are shown in Fig. 7. The larger $L_{3}$ $\mathrm{XMCD}$ peak for $70^{\circ}$ indicates an in-plane easy axis which is confirmed by the steeper slope of the magnetization curve at $\theta=70^{\circ}$ compared to $0^{\circ}$. Again, saturation cannot be reached and the corresponding $\mu_{L} / \mu_{S+7 D}$ value is reported in Table $\mathrm{V}$. The in-plane easy axis is quite unexpected, given that Co adatoms on both Pt(111) (Ref. 4) and Pd(111) (Refs. 73 and 74) present strong out-of-plane anisotropy. The calculations of Sec. III D, however, show that the sign of the MAE of $\mathrm{Co} / \mathrm{Rh}(111)$ can indeed be negative depending on the type of adsorption site (positive for hcp and negative for fcc). Although XMCD measurements cannot distinguish between different absorption sites, these results point out the importance of the substrate and induced magnetization to determine the sign of the MAE.

The average MAE per adatom can be estimated from the magnetization curves shown in Fig. 7(c) for $\mathrm{Co} / \mathrm{Rh}(111)$. The energy $E(\theta, \vartheta, \varphi)$ of a paramagnetic particle of size $s$ being subject to uniaxial magnetic anisotropy $K$ and exposed to an external magnetic field $\mathbf{B}$ is composed of the Zeeman term $-s \mu B \cos \vartheta$ and the MAE term $-s K(\sin \theta \sin \vartheta \cos \varphi$ $+\cos \theta \cos \vartheta)^{2}$. The $z$ axis is defined parallel to the field $\mathbf{B}$, and $\vartheta$ and $\varphi$ are the polar and azimuthal coordinates of the magnetic moment $\mu, \theta$ defines the easy magnetization direction with respect to the surface normal. The mean value of the magnetization is given by Boltzmann statistics and reads
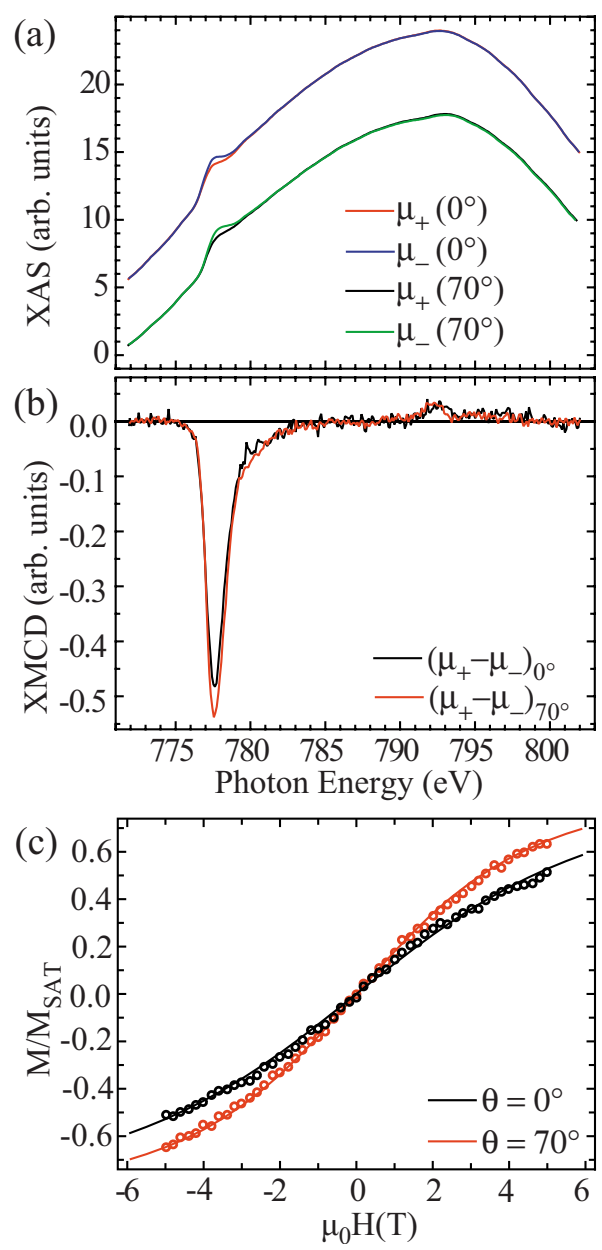

FIG. 7. (Color online) $0.02 \mathrm{ML} \mathrm{Co} / \mathrm{Rh}(111)$ measured at $T$ $=8 \mathrm{~K}$. (a) XAS and (b) XMCD taken at the Co $L_{2,3}$ edges at $B$ $=5 \mathrm{~T}$. Normalization of XAS and XMCD as in Fig. 6. (c) Magnetization curves at $\theta=0^{\circ}$ (black open circles) and $70^{\circ}$ [red (gray) open circles] measured by taking the peak of the $L_{3}$ XMCD intensity at $777.0 \mathrm{eV}$ divided by the pre-edge intensity at $773.5 \mathrm{eV}$ as a function of the applied magnetic field. The solid lines are the best fit obtained for paramagnetic impurities of a mean size $s=1.07$ atoms with uniaxial anisotropy $K=-0.6 \mathrm{meV}$ and a total magnetic moment of $\mu=5 \mu_{B}$.

$$
M=M_{\mathrm{SAT}} \frac{\int_{0}^{2 \pi} d \varphi \int_{0}^{\pi} d \vartheta \sin \vartheta \cos \varphi e^{-E(\theta, \vartheta, \varphi) / k_{B} T}}{\int_{0}^{2 \pi} d \varphi \int_{0}^{\pi} d \vartheta \sin \vartheta e^{-E(\theta, \vartheta, \varphi) / k_{B} T}} .
$$

By fitting this expression to the measured magnetization curves, one obtains the free parameters $\mu, K$, and $M_{\mathrm{SAT}}$. As pointed out before, the signal is dominated by the contribution from single adatoms. However, since the XMCD intensity corresponding to magnetic saturation cannot be determined experimentally in this case, a relatively large uncertainty remains in the fits. Moreover, to avoid increasing the number of fit parameters, no allowance is made for adatoms occupying different adsorption sites. The small amount of dimers is accounted for by using the average size of $s$ $=1.07$ atoms at $0.02 \mathrm{ML}$ coverage. In the case of $\mathrm{Fe} / \mathrm{Pd}(111)$ 
TABLE VI. MAE (in millielectron volt per atom), ratio $\mu_{L} / \mu_{S}$ of orbital and spin magnetic moments and orbital anisotropy for $\mathrm{Fe}$ and $\mathrm{Co}$ adatoms on $\mathrm{Rh}(111), \mathrm{Pd}(111)$, and $\mathrm{Pt}(111)$ substrates. The results for $\mathrm{Rh}$ and Pd substrates are from the present work, those for Pt substrate are from Refs. 4, 21, 73, and 74 (experiment) and Ref. 30 (theory). Where two entries are listed, the first refers to the hcp and the second (in parentheses) to the fcc adsorption site for the adatom.

\begin{tabular}{|c|c|c|c|c|c|}
\hline & \multicolumn{2}{|c|}{ MAE } & \multicolumn{2}{|c|}{$\mu_{L} / \mu_{S}$} & \multirow{2}{*}{$\begin{array}{c}\Delta \mu_{L} \\
\text { Theor. }\end{array}$} \\
\hline & Expt. & Theor. & Expt. $\left(\frac{\mu_{L}}{\mu_{S+7 D}}\right)$ & Theor. $\left(\frac{\mu_{L}}{\mu_{S}}\right)$ & \\
\hline $\mathrm{Fe} / \mathrm{Rh}(111)$ & $\geq 0$ & $0.07(-0.58)$ & $0.15 \pm 0.05$ & 0.03 & $-0.009(-0.010)$ \\
\hline $\mathrm{Fe} / \mathrm{Pd}(111)$ & $\geq 0$ & $-1.8(0.09)$ & $0.12 \pm 0.05$ & 0.02 & $0.000(0.004)$ \\
\hline $\mathrm{Fe} / \mathrm{Pt}(111)$ & $6.5 \pm 0.1$ & 2.99 & & 0.03 & -0.004 \\
\hline $\mathrm{Co} / \mathrm{Rh}(111)$ & $-0.6 \pm 0.1$ & $1.65(-0.29)$ & $0.57 \pm 0.04$ & 0.07 & $-0.006(-0.008)$ \\
\hline $\mathrm{Co} / \mathrm{Pd}(111)$ & $\geq 3$ & $1.61(2.27)$ & $0.70 \pm 0.06$ & 0.10 & $0.032(0.029)$ \\
\hline $\mathrm{Co} / \mathrm{Pt}(111)$ & $9.3 \pm 1.6$ & 1.19 & $0.61 \pm 0.09$ & 0.06 & 0.038 \\
\hline
\end{tabular}

and $\mathrm{Fe} / \mathrm{Rh}(111)$, the difference between out-of-plane and inplane magnetization curves is too small to provide a reliable fit. For $\mathrm{Co} / \mathrm{Rh}(111)$, the best fit is obtained for $\mathrm{MAE}=$ $-0.6 \pm 0.1 \mathrm{meV}$ per atom and $\mu=5 \pm 1 \mu_{B}$ per atom, where $\mu=\mu_{L}+\mu_{S}+\mu_{\text {ind }}(\mathrm{Rh})$ includes the magnetic moment induced on the Rh sites per Co atom. A possibility to derive an experimental estimate of $\mu_{\text {ind }}(\mathrm{Rh})$ is by taking $\mu_{S}$ and $\mu_{S+7 D}$ obtained for the Co monolayer film ${ }^{74}$ and by making use of the fact that $\mu_{S}$ is largely independent of coordination. This way one would obtain an orbital moment of $\mu_{L}=1.2 \mu_{B}$ for the $\mathrm{Co}$ atom and an induced moment of $\mu_{\text {ind }}(\mathrm{Rh})$ $=1.8 \pm 1.0 \mu_{B}$. Such an estimate, however, neglects changes in $\mu_{7 D}$ from the monolayer to the adatom, which can be as large as $0.3 \mu_{B} \cdot{ }^{4}$

\section{DISCUSSION AND CONCLUSIONS}

We have presented a combined theoretical and experimental investigation of orbital magnetism and magnetic anisotropy of single $\mathrm{Fe}$ and $\mathrm{Co}$ adatoms on $\mathrm{Rh}(111)$ and $\mathrm{Pd}(111)$ substrates, complementing earlier results, ${ }^{4,21,30,73,74}$ of the same adatoms on $\mathrm{Pt}(111)$. Table VI confronts the collected experimental and theoretical results.

The experimental determination of the spin and orbital moments and of the MAE using XAS and XMCD spectroscopies turns out to be a very hard task because of the very weak adsorption features in the XAS spectra, the small variation in the magnetization curves with the direction of the applied magnetic field, and finally the difficulty to achieve saturation. For Fe atoms on Pd and Rh weak out-ofplane anisotropy can be inferred from the angular dependence of the $L_{3}$ XMCD peak, and the analysis of the magnetization curves suggests a very low out-of-plane MAE. As saturation cannot be achieved in fields up to $5 \mathrm{~T}$, only the ratio between the effective spin and orbital moments can be determined. For Co on $\mathrm{Rh}(111)$, we find in-plane anisotropy with an MAE of $-0.6 \pm 0.1 \mathrm{meV} /$ atom and significant inplane orbital moment. Fe adatoms on both $\mathrm{Pd}(111)$ and $\mathrm{Rh}$ (111) present significantly weaker orbital-to-spin moment ratios, which, even taking into account the larger spin moment of $\mathrm{Fe}$, indicate a much smaller orbital magnetization relative to Co.
Compared to the large magnetic anisotropies of both elements on $\mathrm{Pt}(111),{ }^{4,21}$ the MAEs of isolated atoms on the (111) surface of the two $4 d$ metals investigated here are much weaker, although the spin and orbital moments of the adatoms are similar. This shows that the magnitude of the MAE is determined primarily by the interaction of the magnetic adatom with the ligands displaying a strong SOC.

The theoretical calculations of the MAE are based on spin-polarized density-functional calculations for slab models of the substrate including a self-consistent treatment of spin-orbit coupling. Self-consistent calculations have been performed for magnetization directions fixed along the surface normal and parallel to the surface of the substrate and the MAE is calculated from the difference in the total energies. In contrast to almost all previous theoretical studies of the MAE of supported nanostructures, our calculations allow for a complete relaxation of the adsorbate/substrate complex. The relaxation effects are particularly pronounced for isolated adatoms which move inward by more than $0.6 \AA$ compared to a position continuing the bulk structure of the substrate. Evidently this leads to a much stronger hybridization between the orbitals of adatom and ligands. We have also determined the energetically most favorable adsorption site and found that a position of the magnetic adatom in an hcp hollow is preferred over the fcc site continuing the stacking sequence of the substrate lattice. We calculated the diffusion barrier limiting the mobility of the adatoms and find that the activation energy for adatom migration is on the order of 0.1 $\mathrm{eV}$. Since the sample is kept at very low temperatures during deposition and measurement, this implies a random distribution of the adatoms over hcp and fcc hollows.

A characteristic property of late transition elements such as $\mathrm{Rh}$ and $\mathrm{Pd}$ is that doping with magnetic impurities induces a large magnetic polarization cloud, the total moment per magnetic atom is very large, substantially larger than the upper limit to the spin moment set by Hund's first rule. For magnetic adatoms on these highly polarizable substrates, the magnetic induction effect is even enhanced by the strong inward relaxation. We have discussed in detail the difficulties arising from these long-range polarization effects in conjunction with a slab model of the substrate. The induced magnetization is not restricted to the formation of an induced spin 
moment - the induced orbital moments are comparable and sometimes even larger than the orbital moments of the adatom. While the magnetic moments of the adatom show only a modest anisotropy, both spin and orbital moments induced in the substrate display a strong anisotropy.

While our calculations yield reasonable values for the MAE (for a detailed discussion, see below), the orbital moments calculated for the adatom are much too low, by a factor of about 6. The reason for this disagreement is twofold: (i) the lack of orbital dependence of the exchange field calculated in DFT and (ii) the strong interaction between adsorbate and ligands atoms which tends to quench the orbital moment to a value comparable to that in bulk materials. In our detailed assessment of different approximations entering the calculation of orbital moments and MAE, illustrated at the example of $\mathrm{Co}$ and $\mathrm{Fe}$ atoms on $\mathrm{Pt}(111),{ }^{30}$ we have demonstrated that for an adatom in an idealized geometry the artificial suppression of the interaction with the ligands allows to form larger orbital moments. But to use a bulklike and, as we have shown, rather unrealistic geometry also strongly reduces the adatom-ligand coupling which is decisive for the magnitude of the MAE. Values of the MAE resulting for both approaches are comparable and tend to be lower than those derived from experiment-indicating that either way theory misses an important contribution to the MAE.

A surprising result of our study is that the MAE is found to be strongly site dependent. Except for $\mathrm{Co} / \mathrm{Pd}(111)$, the MAE changes sign and magnitude if the adatom is placed in an fcc instead of an hep hollow. Since in the experiment, the area covered by the $\mathrm{x}$-ray beam contains many adatoms occupying with comparable probability hep and fcc sites, the experimentally determined MAE must be compared with the average over the MAEs calculated for both sites. The compensation of fcc and hep sites can explain the weak anisotropy observed for $\mathrm{Fe}$ on both substrates. For $\mathrm{Co} / \mathrm{Rh}(111)$, however, we find a positive (out-of-plane) MAE of $1.65 \mathrm{meV}$ and a very modest negative orbital anisotropy for an adatom position in hep hollow sites and a smaller negative (in-plane) MAE of $-0.29 \mathrm{meV}$ and a negative orbital anisotropy for fcc sites. Thus, the arithmetic average of $+0.68 \mathrm{meV}$ disagrees with the experimental value of $-0.6 \mathrm{meV}$, possibly due to uneven distribution of fcc vs hep sites or numerical uncertainties. For $\mathrm{Co} / \mathrm{Pd}(111)$, the calculated positive MAEs of 1.6 $\mathrm{meV}$ (hcp) and $2.3 \mathrm{meV}$ (fcc) indicate strong out-of-plane anisotropy for the ensemble average, in good agreement with experimental estimates. ${ }^{73}$ In these cases, a positive MAE is accompanied by a positive orbital anisotropy on the adatom. This suggest that for sufficiently large MAEs, the sign of the MAE is the same as that of the orbital anisotropy of the magnetic species (as expected on the basis of perturbation theory) while for very small MAEs, this correlation might be broken.

Good semiquantitative agreement between theory and experiment is also found for $\mathrm{Co}$ and $\mathrm{Fe}$ adatoms on $\mathrm{Pt}(111)$. The calculated values for spin and effective (adsorbate plus substrate) moments are in reasonable agreement with experi- ment. The calculated ratios of orbital to spin moment are much too low if orbital polarization is not taken into account. On the experimental side, a precise determination of the orbital moment anisotropy is made difficult by the unknown $\mu_{7 D}$ contribution to the ratio of the orbital to spin moment derived from the XMCD sum rules. ${ }^{71,72,75,76}$ Assuming small $\mu_{7 D}$ anisotropy compared to $\mu_{L}$, the experimental $\mu_{L} / \mu_{S+7 D}$ values would indicate positive orbital moment anisotropy for Fe on $\mathrm{Rh}(111)$ and $\mathrm{Pd}(111)$, as well as for Co on $\mathrm{Pd}(111)$ and $\mathrm{Pt}(111)$ whereas $\mathrm{Co} / \mathrm{Rh}(111)$ would have negative $\Delta \mu_{L}$. The theoretical results agree with these estimates, except for the case of $\mathrm{Fe} / \mathrm{Rh}(111)$, as for the MAE.

We have also attempted to relate the site dependence of the MAE to changes in the electronic structure, using the approximation to the MAE provided by the magnetic force theorem $(\mathrm{MAE}=$ difference in the band energies at fixed potential) and the projected local partial densities of states. This analysis leads to two important conclusions: (i) the contribution of the substrate to the MAE is much more important than estimated before on the basis of calculations using an idealized bulklike geometry. The substrate contribution can even invert the sign of the MAE. (ii) The site dependence is related to a rehybridization of the $d$ states of both adatoms and substrate atoms extending perpendicular to the substrate. (iii) For Co adatoms, the dominant contributions to the MAE arise from a change in occupation of states very close to the Fermi edge. The contribution from the substrate is larger for $\mathrm{Rh}$ than for Pd because of the larger overlap of the $\mathrm{Rh} d$ band with the Co minority states. (iv) The very small MAE of Fe atoms observed on both substrates is related to the large exchange splitting of the Fe $d$ states which minimizes the hybridization of the Fe minority states with the substrate.

In our view, the main problem confronted by $a b$ initio calculations of the MAE is still the lack of orbital dependence of the exchange potential. To use an idealized bulklike and, as we have demonstrated, unrealistic geometry might lead to larger orbital moments. However, this does not represent an acceptable solution because it artificially reduces the adatom/ligand interactions which are the crux of the problem. A fundamental improvement of theoretical predictions of the MAE of supported magnetic nanostructures requires post-DFT corrections introducing the orbital dependence of the exchange potential.

\section{ACKNOWLEDGMENTS}

P.B. and J.H. thank Martijn Marsman for support and fruitful discussions. P.B. and J.H. acknowledge support of the Austrian Science Funds under Project. No. P19712-N16. Financial support from the Swiss National Science Foundation (Grants No. 200020-109800 and No. 200020-112322) and from the European Science Foundation (EUROCORES 05-SONS-FP-009 SANMAG) are gratefully acknowledged. P.G. acknowledges support from the Spanish Ministerio de Ciencia e Innovación (MAT2007-62341) and Agència de Gestió d'Ajuts Universitaris i de Recerca (2009 SGR 695). We thank the ESRF for provision of beam-time. 
${ }^{1}$ M. L. Plumer, J. v. Ek, and D. Weller, in The Physics of Ultrahigh-Density Magnetic Recording, Springer Series in Surface Sciences, edited by G. Ertl, R. Gomer, H. Lüth, and D. L. Mills (Springer, Berlin, 2001), Vol. 41.

${ }^{2}$ D. Sellmyer and R. Skomski, Advanced Magnetic Nanostructures (Springer, New York, 2006).

${ }^{3}$ N. Weiss, T. Cren, M. Epple, S. Rusponi, G. Baudot, S. Rohart, A. Tejeda, V. Repain, S. Rousset, P. Ohresser, F. Scheurer, P. Bencok, and H. Brune, Phys. Rev. Lett. 95, 157204 (2005).

${ }^{4}$ P. Gambardella, S. Rusponi, M. Veronese, S. S. Dhesi, C. Grazioli, A. Dallmeyer, I. Cabria, R. Zeller, P. H. Dederichs, K. Kern, C. Carbone, and H. Brune, Science 300, 1130 (2003).

${ }^{5}$ M. Tischer, O. Hjortstam, D. Arvanitis, J. Hunter Dunn, F. May, K. Baberschke, J. Trygg, J. M. Wills, B. Johansson, and O. Eriksson, Phys. Rev. Lett. 75, 1602 (1995).

${ }^{6}$ J. Stöhr, J. Magn. Magn. Mater. 200, 470 (1999).

${ }^{7}$ P. Gambardella, S. S. Dhesi, S. Gardonio, C. Grazioli, P. Ohresser, and C. Carbone, Phys. Rev. Lett. 88, 047202 (2002).

${ }^{8}$ H. Brune, Surf. Sci. Rep. 31, 125 (1998).

${ }^{9}$ H. Brune and P. Gambardella, Surf. Sci. 603, 1812 (2009).

${ }^{10}$ H. A. Dürr, S. S. Dhesi, E. Dudzik, D. Knabben, G. van der Laan, J. B. Goedkoop, and F. U. Hillebrecht, Phys. Rev. B 59, R701 (1999).

${ }^{11}$ P. Gambardella, A. Dallmeyer, K. Maiti, M. C. Malagoli, W. Eberhardt, K. Kern, and C. Carbone, Nature (London) 416, 301 (2002).

${ }^{12}$ P. Gambardella, A. Dallmeyer, K. Maiti, M. C. Malagoli, S. Rusponi, P. Ohresser, W. Eberhardt, C. Carbone, and K. Kern, Phys. Rev. Lett. 93, 077203 (2004).

${ }^{13}$ T. Koide, H. Miyauchi, J. Okamoto, T. Shidara, A. Fujimori, H. Fukutani, K. Amemiya, H. Takeshita, S. Yuasa, T. Katayama, and Y. Suzuki, Phys. Rev. Lett. 87, 257201 (2001).

${ }^{14}$ G. J. Nieuwenhuys, Adv. Phys. 24, 515 (1975).

${ }^{15}$ Y. Yayon, X. H. Lu, and M. F. Crommie, Phys. Rev. B 73, 155401 (2006).

${ }^{16}$ F. Meier, L. Zhou, J. Wiebe, and R. Wiesendanger, Science 320, 82 (2008).

${ }^{17}$ A. J. Heinrich, J. A. Gupta, C. P. Lutz, and D. M. Eigler, Science 306, 466 (2004).

${ }^{18}$ T. Balashov, A. F. Takács, M. Däne, A. Ernst, P. Bruno, and W. Wulfhekel, Phys. Rev. B 78, 174404 (2008).

${ }^{19}$ C. F. Hirjibehedin, C. Y. Lin, A. F. Otte, M. Ternes, C. P. Lutz, B. A. Jones, and A. J. Heinrich, Science 317, 1199 (2007).

${ }^{20}$ A. F. Otte, M. Ternes, K. v. Bergmann, S. Loth, H. Brune, C. P. Lutz, C. F. Hirjibehedin, and A. J. Heinrich, Nat. Phys. 4, 847 (2008).

${ }^{21}$ T. Balashov, T. Schuh, A. F. Takacs, A. Ernst, S. Ostanin, J. Henk, I. Mertig, P. Bruno, T. Miyamachi, S. Suga, and W. Wulfhekel, Phys. Rev. Lett. 102, 257203 (2009)

${ }^{22}$ O. Eriksson, M. S. S. Brooks, and B. Johansson, Phys. Rev. B 41, 7311 (1990).

${ }^{23}$ D. Fritsch, K. Koepernik, M. Richter, and H. Eschrig, J. Comput. Chem. 29, 2210 (2008).

${ }^{24}$ G. Y. Guo, H. Ebert, W. M. Temmerman, K. Schwarz, and P. Blaha, Solid State Commun. 79, 121 (1991).

${ }^{25}$ L. Szunyogh, B. Ujfalussy, P. Weinberger, and J. Kollár, Phys. Rev. B 49, 2721 (1994).

${ }^{26}$ L. Kleinman, Phys. Rev. B 21, 2630 (1980).

${ }^{27}$ A. H. MacDonald, W. E. Pickett, and D. D. Koelling, J. Phys. C 13, 2675 (1980).
${ }^{28}$ A. R. Mackintosh and O. K. Andersen, in Electrons at the Fermi Surface, edited by M. Springford (Cambridge University Press, London, 1980), Sec. 3.1.

${ }^{29}$ V. Heine, Solid State Phys. 35, 114 (1980).

${ }^{30}$ P. Błoński and J. Hafner, J. Phys.: Condens. Matter 21, 426001 (2009).

${ }^{31}$ S. Bornemann, J. Minar, J. B. Staunton, J. Honolka, A. Enders, K. Kern, and H. Ebert, Eur. Phys. J. D 45, 529 (2007).

${ }^{32}$ O. Šipr, S. Bornemann, J. Minár, S. Polesya, V. Popescu, and A. Šimunek, J. Phys.: Condens. Matter 19, 096203 (2007).

${ }^{33}$ M. Tsujikawa, A. Hosokawa, and T. Oda, J. Phys.: Condens. Matter 19, 365208 (2007).

${ }^{34}$ C. Etz, J. Zabloudil, P. Weinberger, and E. Y. Vedmedenko, Phys. Rev. B 77, 184425 (2008).

${ }^{35}$ A. B. Shick and A. I. Liechtenstein, J. Phys.: Condens. Matter 20, 015002 (2008).

${ }^{36}$ S. Blügel, Phys. Rev. Lett. 68, 851 (1992).

${ }^{37}$ S. Dennler, J. Hafner, M. Marsman, and J. Morillo, Phys. Rev. B 71, 094433 (2005).

${ }^{38}$ D. Spišák and J. Hafner, Phys. Rev. B 67, 214416 (2003).

${ }^{39}$ F. Aguilera-Granja, J. L. Rodriguez-Lopez, K. Michaelian, E. O. Berlanga-Ramirez, and A. Vega, Phys. Rev. B 66, 224410 (2002).

${ }^{40}$ T. Futschek, M. Marsman, and J. Hafner, J. Phys.: Condens. Matter 17, 5927 (2005); 18, 9703 (2006).

${ }^{41}$ J. Honolka, K. Kuhnke, L. Vitali, A. Enders, K. Kern, S. Gardonio, C. Carbone, S. R. Krishnakumar, P. Bencok, S. Stepanow, and P. Gambardella, Phys. Rev. B 76, 144412 (2007).

${ }^{42}$ A. J. Cox, J. G. Louderback, and L. A. Bloomfield, Phys. Rev. Lett. 71, 923 (1993); A. J. Cox, J. G. Louderback, S. E. Apsel, and L. A. Bloomfield, Phys. Rev. B 49, 12295 (1994).

${ }^{43}$ T. Herrmannsdörfer, S. Rehmann, W. Wendler, and F. Pobell, J. Low Temp. Phys. 104, 49 (1996).

${ }^{44}$ K. Swieca, Y. Kondo, and F. Pobell, Phys. Rev. B 56, 6066 (1997).

${ }^{45}$ S. Mitani, K. Takanashi, M. Sano, H. Fujimori, A. Osawa, and H. Nakajima, J. Magn. Magn. Mater. 148, 163 (1995).

${ }^{46}$ G. Kresse and J. Hafner, Phys. Rev. B 47, 558 (1993); 49, 14251 (1994).

${ }^{47}$ J. P. Perdew, J. A. Chevary, S. H. Vosko, K. A. Jackson, M. R. Pederson, D. J. Singh, and C. Fiolhais, Phys. Rev. B 46, 6671 (1992).

${ }^{48}$ S. H. Vosko, L. Wilk, and M. Nusair, Can. J. Phys. 58, 1200 (1980).

${ }^{49}$ E. G. Moroni, G. Kresse, J. Hafner, and J. Furthmüller, Phys. Rev. B 56, 15629 (1997).

${ }^{50}$ G. Kresse and D. Joubert, Phys. Rev. B 59, 1758 (1999).

${ }^{51} \mathrm{G}$. Kresse and O. Lebacq, VASP manual, http:// cms.mpi.univie.ac.at/vasp/

${ }^{52}$ D. Hobbs, G. Kresse, and J. Hafner, Phys. Rev. B 62, 11556 (2000).

${ }^{53}$ M. Marsman and J. Hafner, Phys. Rev. B 66, 224409 (2002).

${ }^{54}$ J. Neugebauer and M. Scheffler, Phys. Rev. B 46, 16067 (1992).

${ }^{55}$ L. Bengtsson, Phys. Rev. B 59, 12301 (1999).

${ }^{56}$ H. J. Monkhorst and J. D. Pack, Phys. Rev. B 13, 5188 (1976).

${ }^{57} \mathrm{http}: / /$ www.webelements.com/

${ }^{58}$ P. Villars and L. D. Calvert, Pearson's Handbook of Crystallographic Data for Intermetallic Phases (ASM, Metals Park, Ohio, 1985).

${ }^{59}$ C. Kittel, Introduction to Solid State Physics, 6th ed. (Wiley, 
New York, 1986)

${ }^{60}$ H. Ohtani, M. A. Van Hove, and G. A. Somorjai, Surf. Sci. 187, 372 (1987).

${ }^{61}$ T. E. Felter, E. C. Sowa, and M. A. Van Hove, Phys. Rev. B 40, 891 (1989).

${ }^{62}$ A. Barbieri, M. A. Van Hove, and G. A. Somorjai, Surf. Sci. 306, 261 (1994).

${ }^{63}$ D. R. Linde, Handbook of Chemistry and Physics, 78th ed. (CRC Press, London, UK, 1998).

${ }^{64}$ S. Schwegmann, H. Over, V. De Renzi, and G. Ertl, Surf. Sci. 375, 91 (1997).

${ }^{65}$ P. Brault, H. Range, J. P. Toennies, and Ch. Wölf, Z. Phys. Chem. (Munich) 198, 1 (1997).

${ }^{66}$ H. Brune, in, Physics of Covered Solid Surfaces, Landolt Börnstein, New Series, Group III Vol. 42, Pt. A, edited by H. P. Bonzel (Springer, Berlin, 2001).

${ }^{67}$ A. Gölzhäuser and G. Ehrlich, Phys. Rev. Lett. 77, 1334 (1996).
${ }^{68}$ P. Bruno, Phys. Rev. B 39, 865 (1989).

${ }^{69}$ C. Andersson, B. Sanyal, O. Eriksson, L. Nordström, O. Karis, D. Arvanitis, T. Konishi, E. Holub-Krappe, and J. Hunter Dunn, Phys. Rev. Lett. 99, 177207 (2007).

${ }^{70}$ G. van der Laan, J. Phys.: Condens. Matter 10, 3239 (1998).

${ }^{71}$ B. T. Thole, P. Carra, F. Sette, and G. van der Laan, Phys. Rev. Lett. 68, 1943 (1992).

${ }^{72}$ P. Carra, B. T. Thole, M. Altarelli, and X. Wang, Phys. Rev. Lett. 70, 694 (1993).

${ }^{73}$ P. Gambardella, A. Vindigni, L. Claude, S. Rusponi, K. Franke, M. Veronese, C. Grazioli, P. Bencok, C. Carbone, and H. Brune (unpublished).

${ }^{74}$ A. Lehnert, Ph.D. thesis, Ecole Polytechnique Fédérale de Lausanne, 2009.

${ }^{75}$ O. Šipr, J. Minár, and H. Ebert, EPL 87, 67007 (2009).

${ }^{76}$ T. Oguchi and T. Shishidou, Phys. Rev. B 70, 024412 (2004). 